Publ. RIMS, Kyoto Univ.

19 (1983), 549-576

\title{
Justification of Partially-Multiplicative Averaging for a Class of Functional-Differential Equations with Impulses
}

\author{
By \\ S. D. Milusheva* and D. D. BAinov**
}

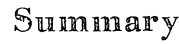

The authors obtain a justification of the method of partially-multiplicative averaging for a class of functional-differential equations with impulses and a transformed argument, dependent on the time and the unknown function.
\end{abstract}

\section{$\S$ H. Introduction}

The averaging method of Bogoljubov-Mitropol'skii is now recognized as one of the most efficient mathematical methods in the nonlinear mechanics. A detailed bibliography on this subject is given in [1]-[3].

In connection with some mathematical models arising in the theory of control systems the averaging method has been justified in [4]-[7] for certain classes of differential equations with impulse action. The generalization of the averaging method for asymptotic integration of systems of differential equations with impulses was substantiated by the following reasons:

-due to their complex structure, the qualitative investigation of the above systems is subject to great difficulties, while the averaged system introduced in the cited papers is without impulse action;

- the solution of the averaged system approximates the solution of the original system with any prescribed accuracy on an asymptotically large time-interval.

The present paper presents a justification of the method of partially-

Communicated by S. Hitotumatu, June 24, 1982.

* Higher Institute of Mechanical and Electrical Engineering, Sofia, Bulgaria.

** University of Plovdiv, Plovdiv, Car Aren 24, Bulgaria. 
multiplicative averaging for a class of functional-differential equations with impulses and a transformed argument dependent on the time and the unknown function.

\section{$\S 2$. Statement of the Problem}

Let in the $(n+1)$-dimensional space $(t, x)$, where $x$ is an $n$-dimensional vector, the following hypersurfaces be given

$$
\sigma_{i}: t=t_{i}(x), i=1,2, \cdots,
$$

which for $x \in D \subset R^{n}$ lie in the half-space $t>0$ and satisfy the condition

$$
t_{i}(x)<t_{i+1}(x), i=1,2, \cdots,
$$

Let a mapping point $P_{t}$ with current coordinates $(t, x(t))$ move in the domain $\{t \geq 0, x \in D\}$. We shall suppose that the motion of the point $P_{t}$ is governed by a law characterized by:

a) the system of differential equations of a neutral type

$$
\begin{aligned}
\dot{x}(t) & =\varepsilon A(t, x(t), x(\Delta(t, x(t))), \dot{x}(\Delta(t, x(t)))) X(t, x(t)), \\
& t>0, \quad t \neq t_{i}(x), \\
x(t) & =\varphi(t, \varepsilon), \quad t \in[-\delta, 0], \\
\dot{x}(t) & =\dot{\varphi}(t, \varepsilon), \quad t \in[-\delta, 0],
\end{aligned}
$$

where $\varepsilon$ is a small parameter, $A(t, x, y, z)=\left(a_{i j}(t, x, y, z)\right)_{n m}, \delta$ is a positive constant, $\Delta(t, x)$ is a transformed argument satisfying the condition

$$
t-\delta \leq \Delta(t, x) \leq t
$$

for $t \geq-\delta$ and $x \in D$, and $\varphi(t, \varepsilon)$ is an initial-value function defined together with its derivative $\dot{\varphi}(t, \varepsilon)$ with respect to $t$ for $t \in[-\delta, 0]$ and $\varepsilon \in(0, \mathcal{E}], \varepsilon=\mathrm{const}>0$;

b) the set of hypersurfaces $\sigma_{i}, \quad i=1,2, \cdots$;

c) the set of vector-functions $I_{i}(x), \quad i=1,2, \cdots$, defined in $D$.

Note that in view of (2) the velocity of the point $P_{t}$ at time $t$ depends on the motion and velocity of $P_{t}$ on the whole preceding interval $[t-\delta, t]$. 
The motion itself can be described as follows. Departing from the point $\left(\tau_{0}=0, x_{0}=x(0)=\varphi(0, \varepsilon)\right)$ the point $P_{t}$ moves along the trajectory $(t, x(t))$, governed by the solution $x(t)$ of (1) until the moment $\tau_{1}>0$ at which the trajectory meets the hypersurface $\sigma_{1}$ at the point $\left(\tau_{1}, x_{1}^{-}\right.$ $\left.=x\left(\tau_{1}\right)\right)$. Then the point $P_{t}$ instantly moves from the position $\left(\tau_{1}, x_{1}^{-}\right)$ to the position $\left(\tau_{1}, x_{1}^{+}=x_{1}^{-}+\varepsilon I_{1}\left(x_{1}^{-}\right)\right)$and further on follows the trajectory $(t, x(t))$, described by the solution $x(t)$ of system (1) until it meets the hypersurface $\sigma_{2}$, etc.

The relations a), b), c) characterizing the motion of point $P_{t}$ are said to be a system of functional-differential equations (1) with impulses. The curve described by the motion of point $P_{t}$ is said to be the integral curve or the trajectory of this system in the space $(t, x)$.

Thus the solution of the system of functional-differential equations (1) with impulses is a function satisfying (1) out of the hypersurfaces $\sigma_{i}, i=1,2, \cdots$ and having instantaneous jumps

$$
x_{i}^{+}=x_{i}^{-}+\varepsilon I_{i}\left(x_{i}^{-}\right), \quad i=1,2, \cdots
$$

when meeting the hypersurfaces $\sigma_{i}, i=1,2, \cdots$. Note that the point $\left(\tau_{i}, x_{i}^{+}\right)$does not necessarily belong to the hypersurface $\sigma_{i}, i=1,2, \cdots$.

Let the following limits exist

$$
\begin{aligned}
& \lim _{T \rightarrow \infty} \frac{1}{T} \int_{t}^{t+T} A(\theta, x, x, 0) d \theta=A_{0}(x) \\
& \lim _{T \rightarrow \infty} \frac{1}{T} \sum_{t<t_{i}<t+T} I_{i}(x)=I_{0}(x) .
\end{aligned}
$$

Then we compare the system of functicnal-differential equations (1) with impulses to the averaged system of ordinary differential equations

$$
\dot{\bar{x}}(t)=\varepsilon\left[A_{0}(\bar{x}(t)) X(t, \bar{x}(t))+I_{0}(\bar{x}(t))\right]
$$

with initial condition

$$
\bar{x}(0)=x_{0} .
$$

Note that if $x=\left(x_{1}, \cdots, x_{n}\right), A=\left(a_{i j}\right)_{n m}$, then by definition

$$
\|x\|=\left[\sum_{i=1}^{n} x_{i}^{2}\right]^{1 / 2},\|A\|=\left[\sum_{i=1}^{n} \sum_{j=1}^{m} a_{i j}^{2}\right]^{1 / 2},
$$

while by $\overline{1, n}$ we shall denote the set of positive integers $\{1,2, \cdots, n\}$. 


\section{$\S 3 . \quad$ Main Result}

The following theorem for proximity between the solutions of the system of functional-differential equations (1) with impulses and the averaged system (5) with initial condition (6) holds true:

Theorem 1. Let the following assumptions be fulfilled:

$1^{\circ}$ The functions $A(t, x, y, z)$ and $X(t, x)$ are continuous in the comain $\left\{t \geq 0, x, y \in D, \approx \in D_{1} \subset R^{n}\right\}$. The function $\Delta(t, x)$ is continuous and satisfies the condition (2) in the domain $\{t \geq 0, x \in D\}$. The functions $\varphi(t, \varepsilon)$ and $\dot{\varphi}(t, \varepsilon)$ are continuous in the domain $\{t \in[-\delta, 0]$, $\varepsilon \in(0, \mathcal{E}], \mathcal{E}=\mathrm{const}>0\}$ and $\varphi(t, \varepsilon) \in D, \dot{\varphi}(t, \varepsilon) \in D_{1}$. The functions $I_{i}(x), i=1,2, \cdots$ are continuous in $D$. The functions $t_{i}(x), i=1,2, \cdots$ are twice continuously differentiable in $D$.

$2^{\circ}$ There exist positive constants $M, K, C$ and a function $r(\varepsilon)$ such that

$$
\begin{aligned}
& \left\|\frac{\partial t_{i}(x)}{\partial x}\right\|+\|A(t, x, y, z)\|+\|X(t, x)\|+\left\|I_{i}(x)\right\| \leq M \\
& \left\|A(t, x, y, \approx)-A\left(t, x^{\prime}, y^{\prime}, z^{\prime}\right)\right\| \leq K\left(\left\|x-x^{\prime}\right\|+\left\|y-y^{\prime}\right\|+\left\|z-z^{\prime}\right\|\right), \\
& \left\|X(t, x)-X\left(t, x^{\prime}\right)\right\|+\left\|I_{i}(x)-I_{i}\left(x^{\prime}\right)\right\| \leq K\left\|x-x^{\prime}\right\|,\left\|\frac{\partial^{2} t_{i}(x)}{\partial x^{2}}\right\| \leq C
\end{aligned}
$$

for all $t \geq 0, x, x^{\prime}, y, y^{\prime} \in D, \approx, z^{\prime} \in D_{1}, i=1,2, \cdots$ and $\|\dot{\varphi}(t, \varepsilon)\| \leq r(\varepsilon)$ for $\varepsilon \in(0, \varepsilon]$, where $\lim _{\varepsilon \rightarrow 0}(\gamma(\varepsilon) / \varepsilon)=\mathrm{const}>0$ and $\sup _{\varepsilon \in(0, \varepsilon]}(\gamma(\varepsilon) / \varepsilon)=\mathrm{const}>0$.

$3^{\circ}$ Uniformly in $t \geq 0$ and $x \in D$ there exist the finite limits (4) and

$$
\lim _{T \rightarrow \infty} \frac{1}{T} \sum_{t<t_{i}<t+T} 1=d, \quad d=\mathrm{const}>0
$$

$4^{\circ}$ The functions $a_{i j}(t, x, y, z)-a_{i j}^{(0)}(x), i=\overline{1, n}, j=\overline{1, m}$, where $a_{i j}^{(0)}$ are the elements of the matrix $A_{0}(x)$, do not change sign in the whole domain $\left\{t \geq 0, x, y \in D, z \in D_{1}\right\}$, i.e. either $a_{i j}(t, x, y, z)-a_{i j}^{(0)}(x)$ $\geq 0$ or $a_{i j}(t, x, y, \approx)-a_{i j}^{(0)}(x) \leq 0$ in this domain.

$5^{\circ}$ For each $\varepsilon \in(0, \varepsilon]$ the syslem of Junctional-differential equations (1) with impulses has a continuous solution $x(t)$ for $t \geq 0$, 
$t \neq \tau_{i}, \quad i=1,2, \cdots$ rohich salisfics the matching conditions $x(0+0)=$ $\varphi(0, \varepsilon)=x_{0}, \dot{x}(0+0)=\dot{\varphi}(0, \varepsilon)$.

$6^{\circ}$ For each $\varepsilon \in(0, \varepsilon]$ the averaged initial value problem (5), (6) has a solution $\bar{x}(t)$, which belongs to the domain $D$ for $t \geq 0$ together rith its neighbourhood of radius $\rho=\mathrm{const}>0$, and satisfies the inequalities $\frac{\partial t_{i}(\bar{x}(t))}{\partial x} I_{i}(\bar{x}(t)) \leq \beta<0, \beta=$ const, $t \in\left(t_{i}^{\prime}, t_{i}^{\prime \prime}\right), t_{i}^{\prime}=\inf _{x \in D} t_{i}(x)$, $t_{i}^{\prime \prime}=\sup _{x \in D} t_{i}(x), i=1,2, \cdots$, or $\frac{\partial t_{i}(x)}{\partial x} \equiv 0$, when $\sigma_{i}$ is a hyperplane.

Then for each $\eta>0$ and $L>0$ there exists $\varepsilon_{0} \in(0, \varepsilon]\left(\varepsilon_{0}=\varepsilon_{0}(\eta, L)\right)$ such that for $\varepsilon \leq \varepsilon_{0}$ the inequality $\|x(t)-\bar{x}(t)\|<\eta$ holds for $0 \leq l$ $\leq L \varepsilon^{-1}$.

We shall base the proof of Theorem 1 on the following lemma.

Lemma 1. Let the conditions of Theorem 1 be fulfilled. Let $T>\delta$ be a sufficiently large and fixed number. Then for each positive integer $p \geq 1$ the follorving inequality holds

$$
\begin{aligned}
& \|x(p T)-\bar{x}(p T)\| \\
& \quad \leq \varepsilon \sum_{i=0}^{p-1}[1+\varepsilon(3 M+d) K T]^{i}[\alpha(T) T+\varepsilon \bar{M}],
\end{aligned}
$$

rohere $\bar{M}=(M+d)(3 M+d) K M T^{2}+\max _{i=1, p} M_{i}$ and $M_{i}=M_{i}\left(T, d_{1}, \cdots, d_{i}\right)$ are constants depending on $T$ and on the constants $d_{j}>0, j=\overline{1, i}$.

Proof of Lemma 1. The condition $3^{\circ}$ of Theorem 1 guarantees the cxistence of a function $\alpha(t)$, monotonously decreasing towards zero as $\iota$ tends to infinity, such that for each $l \geq 0$ and $x \in D$ the following inequalities hold

$$
\begin{aligned}
& \text { 1) } \int_{t}^{t+T}\left[A(\theta, x, x, 0)-A_{0}(x)\right] d \theta \mid \leq \alpha(T) T / 2 M m \sqrt{n}, \\
& \left\|\sum_{t<t_{i}<t+T} I_{i}(x)-I_{0}(x) T\right\| \leq \alpha(T) T / 2 .
\end{aligned}
$$

We shall carry out the proof of Lemma 1 by the method of complete mathematical induction.

First we shall prove the inequality (7) for $p=1$. 
We consider the system of functional-differential equations (1) with impulses on the interval $[0, T]$.

Let $d_{1}$ points lie on the interval $(0, T)$

$$
t_{1}\left(x_{0}\right)=t_{1}^{(0)}, \cdots, t_{d_{1}}\left(x_{0}\right)=t_{d_{1}}^{(0)}
$$

in which case $t_{i}^{(0)}<t_{i+1}^{(0)}, i=\overline{1,\left(d_{1}-1\right)}$.

We denote by $x_{1}^{(0)}\left(t, 0, x_{0}\right)$ the sclution of the system

$$
\begin{aligned}
(10) \quad x_{1}^{(0)}\left(t, 0, x_{0}\right) & =\left\{\begin{array}{l}
x_{0}+\varepsilon \int_{0}^{t} A_{0}\left(\theta, x_{1}^{(0)}\left(\theta, 0, x_{0}\right), x_{1}^{(0)}\left(\Delta_{1}^{(0)}(\theta), 0, x_{0}\right),\right. \\
\left.\dot{x}_{1}^{(0)}\left(\Delta_{1}^{(0)}(\theta), 0, x_{0}\right)\right) X\left(\theta, x_{1}^{(0)}\left(\theta, 0, x_{0}\right)\right) d \theta, \quad t>0 . \\
\varphi(t, \varepsilon), \quad-\delta \leq t \leq 0,
\end{array}\right. \\
\dot{x}_{1}^{(0)}\left(t, 0, x_{0}\right) & =\dot{\varphi}(t, \varepsilon), \quad-\delta \leq t \leq 0,
\end{aligned}
$$

where $\Delta_{1}^{(0)}(t)=\Delta\left(t, x_{1}^{(0)}\left(t, 0, x_{0}\right)\right)$.

Obviously, the solution of (10) coincides with the solution $x(t)$ of the system of functional-differential equations (1) with impulses until the moment $\tau_{1}$ at which the trajectory $(t, x(t))$ of this system meets the hypersurface $\sigma_{1}$, i.e. $x(t)=x_{1}^{(0)}\left(t, 0, x_{0}\right), \mathrm{t} \in\left[-\delta, \tau_{1}\right]$.

Let us consider the function

$$
\widetilde{x}_{1}^{(0)}\left(t, 0, x_{0}\right)=x_{0}+\varepsilon \int_{0}^{t} A\left(\theta, x_{0}, x_{0}, 0\right) X\left(\theta, x_{0}\right) d \theta
$$

and estimate in terms of norm the difference

$$
R_{1}^{(0)}\left(t, 0, x_{0}, \varepsilon\right)=x_{1}^{(0)}\left(t, 0, x_{0}\right)-\widetilde{x}_{1}^{(0)}\left(t, 0, x_{0}\right) .
$$

For $0<t \leq T$ we have

$$
\begin{aligned}
& \left\|R_{1}^{(0)}\left(t, 0, x_{0}, \varepsilon\right)\right\| \leq \varepsilon \int_{0}^{t} \| A\left(\theta, x_{1}^{(0)}\left(\theta, 0, x_{0}\right), x_{1}^{(0)}\left(\Delta_{1}^{(0)}(\theta), 0, x_{0}\right),\right. \\
& \left.\quad \dot{x}_{1}^{(0)}\left(\Delta_{1}^{(0)}(\theta), 0, x_{0}\right)\right) X\left(\theta, x_{1}^{(0)}\left(\theta, 0, x_{0}\right)\right)-A\left(\theta, x_{0}, x_{0}, 0\right) X\left(\theta, x_{0}\right) \| d \theta \\
& \leq \varepsilon \int_{0}^{t}\left\{\| A\left(\theta, x_{1}^{(0)}\left(\theta, 0, x_{0}\right), x_{1}^{(0)}\left(\Delta_{1}^{(0)}(\theta), 0, x_{0}\right), \dot{x}_{1}^{(0)}\left(\Delta_{1}^{(0)}(\theta), 0, x_{0}\right)\right)\right. \\
& \quad-A\left(\theta, x_{0}, x_{0}, 0\right)\|\cdot\| X\left(\theta, x_{1}^{(0)}\left(\theta, 0, x_{0}\right)\right)\|+\| A\left(\theta, x_{0}, x_{0}, 0\right) \| \\
& \left.\cdot\left\|X\left(\theta, x_{1}^{(0)}\left(\theta, 0, x_{0}\right)\right)-X\left(\theta, x_{0}\right)\right\|\right\} d \theta \leq \varepsilon K M \int_{0}^{t}\left\{2\left\|x_{1}^{(0)}\left(\theta, 0, x_{0}\right)-x_{0}\right\|\right. \\
& \left.\quad+\left\|x_{1}^{(0)}\left(\Delta_{1}^{(0)}(\theta), 0, x_{0}\right)-x_{0}\right\|+\left\|\dot{x}_{1}^{(0)}\left(\Delta_{1}^{(0)}(\theta), 0, x_{0}\right)\right\|\right\} d \theta
\end{aligned}
$$




$$
\begin{aligned}
& \leq 2 \varepsilon^{2} K M \int_{0}^{t} d \theta \int_{0}^{\theta} \| A\left(l, x_{1}^{(0)}\left(l, 0, x_{0}\right), x_{1}^{(0)}\left(\Delta_{1}^{(0)}(l), 0, x_{0}\right),\right. \\
& \left.\dot{x}_{1}^{(0)}\left(\Delta_{1}^{(0)}(l), 0, x_{0}\right)\right)\|\cdot\| X\left(l, x_{1}^{(0)}\left(l, 0, x_{0}\right)\right) \| d l \\
& +\varepsilon K M\left\{\int_{J_{0, \tau}^{-}}\left\|\varphi\left(\Delta_{1}^{(0)}(\theta), \varepsilon\right)-\varphi(0, \varepsilon)\right\| d \theta\right. \\
& +\varepsilon \int_{J_{0, t}^{+}} d \theta \int_{0}^{\Delta_{1}^{(0)}(\theta)} \| A\left(l, x_{1}^{(0)}\left(l, 0, x_{0}\right),\right. \\
& \left.\left.x_{1}^{(0)}\left(\Delta_{1}^{(0)}(l), 0, x_{0}\right), \dot{x}_{1}^{(0)}\left(\Delta_{1}^{(0)}(l), 0, x_{0}\right)\right)\|\cdot\| X\left(l, x_{1}^{(0)}\left(l, 0, x_{0}\right)\right) \| d l\right\} \\
& +\varepsilon K M\left\{\int_{J_{0, t}^{-}}\left\|\dot{\varphi}\left(\Delta_{1}^{(0)}(\theta), \varepsilon\right)\right\| d \theta\right. \\
& +\varepsilon \int_{J_{0, t}^{+}} \| A\left(\Delta_{1}^{(0)}(\theta), x_{1}^{(0)}\left(\Delta_{1}^{(0)}(\theta), 0, x_{0}\right),\right. \\
& \left.\left.x_{1}^{(0)}\left(\Delta_{1}^{(0)}\left(\Delta_{1}^{(0)}(\theta)\right), 0, x_{0}\right), \dot{x}_{1}^{(0)}\left(\Delta_{1}^{(0)}(\theta)\right), 0, x_{0}\right)\right)\|\cdot\| X\left(\Delta_{1}^{(0)}(\theta),\right. \\
& \left.\left.x_{1}^{(0)}\left(\Delta_{1}^{(0)}(\theta), 0, x_{0}\right)\right) \| d \theta\right\} \leq 2 \varepsilon^{2} K M^{3} \int_{0}^{t} d \theta \int_{0}^{\theta} d l \\
& +\varepsilon \gamma(\varepsilon) \sqrt{n} K M \int_{J_{0, \tau}^{-}}\left|\Delta_{1}^{(0)}(\theta)\right| d \theta+\varepsilon^{2} K M^{3} \int_{J_{0, t}^{+}} \Delta_{1}^{(0)}(\theta) d \theta \\
& +\varepsilon \gamma(\varepsilon) K M \int_{J_{0, t}^{-}} d \theta+\varepsilon^{2} K M^{3} \int_{J_{0, t}^{+}} d \theta \leq \varepsilon^{2} K M^{3} T^{2} \\
& +\varepsilon \gamma(\varepsilon)(\delta \sqrt{n}+1) K M \int_{0}^{t} d \theta+\varepsilon^{2} K M^{3} \int_{0}^{t} \theta d \theta+\varepsilon^{2} K M^{3} \int_{0}^{t} d \theta \\
& \leq 3 \varepsilon^{2} K M^{3} T^{2} / 2+\varepsilon \gamma(\varepsilon)(\delta \sqrt{n}+1) K M T+\varepsilon^{2} K M^{3} T \equiv \omega_{1}^{(0)}\left(\varepsilon^{2}, T\right),
\end{aligned}
$$

where

$$
\begin{aligned}
& J_{0, t}^{-} \cup J_{0, t}^{+}=(0, t], \\
& J_{0, t}^{-}=\left\{\theta: \theta \in(0, t] \wedge \Delta_{1}^{(0)}(\theta) \in[-\delta, 0]\right\}, \\
& J_{0, t}^{+}=(0, t] \backslash J_{0, t}^{-} .
\end{aligned}
$$

The obtained estimate shows that the function $\widetilde{x}_{1}^{(0)}\left(t, 0, x_{0}\right)$ approximates the solution $x_{1}^{(0)}\left(t, 0, x_{0}\right)$ of the system (10) on the interval $(0, T]$ to a precision of order $\varepsilon^{2}$.

The moment $\tau_{1}$, at which the trajectory $(t, x(t))$ meets the hypersurface $\sigma_{1}$, is a solution of the equation

$$
t=t_{1}\left(x_{1}^{(0)}\left(t, 0, x_{0}\right)\right) .
$$


Since

$$
\begin{aligned}
& t_{1}\left(x_{1}^{(0)}\left(t, 0, x_{0}\right)\right)=t_{1}\left(\widetilde{x}_{1}^{(0)}\left(t, 0, x_{0}\right)+R_{1}^{(0)}\left(t, 0, x_{0}, \varepsilon\right)\right) \\
& =t_{1}\left(x_{0}+\varepsilon \int_{0}^{t} A\left(\theta, x_{0}, x_{0}, 0\right) X\left(\theta, x_{0}\right) d \theta+O\left(\varepsilon^{2}\right)\right) \\
& =t_{1}\left(x_{0}\right)+\varepsilon \frac{\partial t_{1}\left(x_{0}\right)}{\partial x} \int_{0}^{t} A\left(\theta, x_{0}, x_{0}, 0\right) X\left(\theta, x_{0}\right) d \theta+O\left(\varepsilon^{2}\right) \\
& =t_{1}^{(0)}+\varepsilon \frac{\partial t_{1}\left(x_{0}\right)}{\partial x} \int_{0}^{t_{1}^{(0)}} A\left(\theta, x_{0}, x_{0}, 0\right) X\left(\theta, x_{0}\right) d \theta \\
& \quad+\varepsilon \frac{\partial t_{1}\left(x_{0}\right)}{\partial x} \int_{t_{1}^{(0)}}^{t} A\left(\theta, x_{0}, x_{0}, 0\right) X\left(\theta, x_{0}\right) d \theta+O\left(\varepsilon^{2}\right) \\
& =t_{1}^{(0)}+\varepsilon \frac{\partial t_{1}\left(x_{0}\right)}{\partial x} \int_{0}^{t_{1}^{(0)}} A\left(\theta, x_{0}, x_{0}, 0\right) X\left(\theta, x_{0}\right) d \theta \\
& \quad+\varepsilon \frac{\partial t_{1}\left(x_{0}\right)}{\partial x}\left(t-t_{1}^{(0)}\right) A\left(\tilde{t}, x_{0}, x_{0}, 0\right) X\left(\tilde{t}, x_{0}\right)+O\left(\varepsilon^{2}\right), \\
& \tilde{t}=t_{1}^{(0)}+\mu\left(t-t_{1}^{(0)}\right), 0 \leq \mu \leq 1,
\end{aligned}
$$

then from (11) it follows that $\tau_{1}=t_{1}^{(0)}+\varepsilon \Theta_{1}^{(0)}+O\left(\varepsilon^{2}\right)$, where

$$
\Theta_{1}^{(0)}=\frac{\partial t_{1}\left(x_{0}\right)}{\partial x} \int_{0}^{t_{1}^{(0)}} A\left(\theta, x_{0}, x_{0}, 0\right) X\left(\theta, x_{0}\right) d \theta
$$

We shall note that in (12) the values of the constant $\mu$ in the different components of the vector $A\left(\tilde{t}, x_{0}, x_{0}, 0\right) X\left(\tilde{t}, x_{0}\right)$ are, generally speaking, different.

The inequality $t_{1}^{(0)}>0$ implies that $\tau_{1}>\tau_{0}$ if $\varepsilon$ is sufficiently small.

Thus

$$
x(t)=x_{1}^{(0)}\left(t, 0, x_{0}\right)=\tilde{x}_{1}^{(0)}\left(t, 0, x_{0}\right)+R_{1}^{(0)}\left(t, 0, x_{0}, \varepsilon\right)
$$

for $\tau_{0}<t \leq \tau_{1}=t_{1}^{(0)}+\varepsilon \Theta_{1}^{(0)}+O\left(\varepsilon^{2}\right)$.

Henceforth we find

$$
x_{1}^{+}=x_{1}^{(0)}\left(\tau_{1}, 0, x_{0}\right)+\varepsilon I_{1}\left(x_{1}^{(0)}\left(\tau_{1}, 0, x_{0}\right)\right)
$$

i.e.

$$
\begin{aligned}
x_{1}^{+} & =\widetilde{x}_{1}^{(0)}\left(\tau_{1}, 0, x_{0}\right)+\varepsilon I_{1}^{(0)}+R_{1}^{(0)}\left(\tau_{1}, 0, x_{0}, \varepsilon\right) \\
& =x_{0}+\varepsilon \int_{0}^{\tau_{1}} A\left(\theta, x_{0}, x_{0}, 0\right) X\left(\theta, x_{0}\right) d \theta+\varepsilon I_{1}^{(0)}+R_{1}^{(0)}\left(\tau_{1}, 0, x_{0}, \varepsilon\right),
\end{aligned}
$$


where $I_{1}^{(0)} \equiv I_{1}\left(x_{1}^{(0)}\left(\tau_{1}, 0, x_{0}\right)\right)$.

We denote by $x_{2}^{(0)}\left(t, \tau_{1}, x_{1}^{+}\right)$the solution of the system

$$
\begin{aligned}
& x_{2}^{(0)}\left(t, \tau_{1}, x_{1}^{+}\right)=\left\{\begin{array}{l}
x_{1}^{+}+\varepsilon \int_{\tau_{1}}^{t} A\left(\theta, x_{2}^{(0)}\left(\theta, \tau_{1}, x_{1}^{+}\right), x_{2}^{(0)}\left(\Delta_{2}^{(0)}(\theta), \tau_{1}, x_{1}^{+}\right),\right. \\
\left.\dot{x}_{2}^{(0)}\left(\Delta_{2}^{(0)}(\theta), \tau_{1}, x_{1}^{+}\right)\right) X\left(\theta, x_{2}^{(0)}\left(\theta, \tau_{1}, x_{1}^{+}\right)\right) d \theta, \quad t>\tau_{1}, \\
x_{1}^{(0)}\left(t, 0, x_{0}\right), \quad-\delta \leq t \leq \tau_{1},
\end{array}\right. \\
& \dot{x}_{2}^{(0)}\left(t, \tau_{1}, x_{1}^{+}\right)=\dot{x}_{1}^{(0)}\left(t, 0, x_{0}\right), \quad-\delta \leq t \leq \tau_{1},
\end{aligned}
$$

where $\Delta_{2}^{(0)}(t)=\Delta\left(t, x_{2}^{(0)}\left(t, \tau_{1}, x_{1}^{+}\right)\right)$.

The solution of (13) coincides with the solution $x(t)$ of the system of functional-differential equations (1) with impulses until the moment $\tau_{2}$, at which the trajectory $(t, x(t))$ meets the hypersurface $\sigma_{2}$, i.e.

$$
x(t)=x_{2}^{(0)}\left(t, \tau_{1}, x_{1}^{+}\right) \quad \text { for } \quad t \in\left[-\delta, \tau_{2}\right] .
$$

Let us consider the function

$$
\widetilde{x}_{2}^{(0)}\left(t, \tau_{1}, x_{1}^{+}\right)=x_{1}^{+}+\varepsilon \int_{\tau_{1}}^{t} A\left(0, x_{0}, x_{0}, 0\right) X\left(\theta, x_{0}\right) d 0
$$

and estimate in terms of norm the difference

$$
R_{2}^{(0)}\left(t, \tau_{1}, x_{1}^{+}, \varepsilon\right)=x_{2}^{(0)}\left(t, \tau_{1}, x_{1}^{+}\right)-\widetilde{x}_{2}^{(0)}\left(t, \tau_{1}, x_{1}^{+}\right) .
$$

For $0<\tau_{1}<t \leq T$ we obtain

$$
\begin{aligned}
& \left\|R_{2}^{(0)}\left(t, \tau_{1}, x_{1}^{+}, \varepsilon\right)\right\| \leq \varepsilon \int_{\tau_{1}}^{t} \| A\left(\theta, x_{2}^{(0)}\left(\theta, \tau_{1}, x_{1}^{+}\right), x_{2}^{(0)}\left(\Delta_{2}^{(0)}(\theta), \tau_{1}, x_{1}^{+}\right),\right. \\
& \left.\quad \dot{x}_{2}^{(0)}\left(\Delta_{2}^{(0)}(\theta), \tau_{1}, x_{1}^{+}\right)\right) X\left(\theta, x_{2}^{(0)}\left(\theta, \tau_{1}, x_{1}^{+}\right)\right)-A\left(\theta, x_{0}, x_{0}, 0\right) X\left(\theta, x_{0}\right) \| d \theta \\
& \leq \varepsilon \int_{\tau_{1}}^{t}\left\{\| A\left(\theta, x_{2}^{(0)}\left(\theta, \tau_{1}, x_{1}^{+}\right), x_{2}^{(0)}\left(\Delta_{2}^{(0)}(\theta), \tau_{1}, x_{1}^{+}\right), \dot{x}_{2}^{(0)}\left(\Delta_{2}^{(0)}(\theta), \tau_{1}, x_{1}^{+}\right)\right)\right. \\
& \quad-A\left(\theta, x_{0}, x_{0}, 0\right)\|\cdot\| X\left(\theta, x_{2}^{(0)}\left(\theta, \tau_{1}, x_{1}^{+}\right)\right)\|+\| A\left(\theta, x_{0}, x_{0}, 0\right) \| \\
& \left.\quad \cdot\left\|X\left(0, x_{2}^{(0)}\left(\theta, \tau_{1}, x_{1}^{+}\right)\right)-X\left(\theta, x_{0}\right)\right\|\right\} d 0 \\
& \leq \varepsilon K M \int_{\tau_{1}}^{t}\left\{2\left\|x_{2}^{(0)}\left(\theta, \tau_{1}, x_{1}^{+}\right)-x_{0}\right\|+\left\|x_{2}^{(0)}\left(\Delta_{2}^{(0)}(\theta), \tau_{1}, x_{1}^{+}\right)-x_{0}\right\|\right. \\
& \left.\left.\quad+\| \dot{x}_{2}^{(0)}\left(\Delta_{2}^{(0)}(\theta), \tau_{1}, x_{1}^{+}\right)\right) \|\right\} d \theta \leq 2 \varepsilon^{2} K M I \int_{\tau_{1}}^{t}\left\{\int_{0}^{\tau_{1}}\left\|A\left(l, x_{0}, x_{0}, 0\right)\right\|\right. \\
& \quad \cdot\left\|X\left(l, x_{0}\right)\right\| d l+\left\|I_{1}^{(0)}\right\|+\varepsilon^{-1}\left\|R_{1}^{(0)}\left(\tau_{1}, 0, x_{0}, \varepsilon\right)\right\|
\end{aligned}
$$




$$
\begin{aligned}
& +\int_{\tau_{1}}^{\theta}\left\|A\left(l, x_{2}^{(0)}\left(l, \tau_{1}, x_{1}^{+}\right), x_{2}^{(0)}\left(\Delta_{2}^{(0)}(l), \tau_{1}, x_{1}^{-}\right), \dot{x}_{2}^{(0)}\left(\Delta_{2}^{(0)}(l), \tau_{1}, x_{1}^{+}\right)\right)\right\| \\
& \text {- } \left.\left\|X\left(l, x_{2}^{(0)}\left(l, \tau_{1}, x_{1}^{+}\right)\right)\right\| d l\right\} d \theta+\varepsilon K M\left\{\int_{J_{\tau_{1}}^{-}, t}\left\|\varphi\left(\Delta_{2}^{(0)}(\theta), \varepsilon\right)-\varphi(0, \varepsilon)\right\| d \theta\right. \\
& +\int_{J_{\tau_{1}}, t}\left\|x_{1}^{(0)}\left(\Delta_{2}^{(0)}(\theta), 0, x_{0}\right)-x_{0}\right\| d \theta+\varepsilon \int_{J_{\tau_{1}, t}^{+}}\left[\int_{0}^{\tau_{1}}\left\|A\left(l, x_{0}, x_{0}, 0\right)\right\|\right. \\
& \cdot\left\|X\left(l, x_{0}\right)\right\| d l+\left\|I_{1}^{(0)}\right\|+\varepsilon^{-1}\left\|R_{1}^{(0)}\left(\tau_{1}, 0, x_{0}, \varepsilon\right)\right\| \\
& +\int_{\tau_{1}}^{\Delta_{2}^{(0)}(\theta)} \| A\left(l, x_{2}^{(0)}\left(l, \tau_{1}, x_{1}^{+}\right), x_{2}^{(0)}\left(\Delta_{2}^{(0)}(l), \tau_{1}, x_{1}^{+}\right),\right. \\
& \left.\left.\left.\dot{x}_{2}^{(0)}\left(\Delta_{2}^{(0)}(l), \tau_{1}, x_{1}^{+}\right)\right)\|\cdot\| X\left(l, x_{2}^{(0)}\left(l, \tau_{1}, x_{1}^{+}\right)\right) \| d l\right] d \theta\right\} \\
& +\varepsilon K M\left\{\int_{J_{\tau_{1}}^{-}, t}\left\|\dot{\varphi}\left(\Delta_{2}^{(0)}(\theta)\right)\right\| d \theta+\varepsilon \int_{J_{\tau_{1}, t}^{1}} \| A\left(\Delta_{2}^{(0)}(\theta), x_{1}^{(0)}\left(\Delta_{2}^{(0)}(\theta), 0, x_{0}\right),\right.\right. \\
& x_{1}^{(0)}\left(\Delta_{1}^{(0)}\left(\Delta_{2}^{(0)}(\theta)\right), 0, x_{0}\right), \dot{x}_{1}^{(0)}\left(\Delta_{1}^{(0)}\left(\Delta_{2}^{(0)}(\theta)\right), 0, x_{0}\right) \| \\
& \text { - }\left\|X\left(\Delta_{2}^{(0)}(\theta), x_{1}^{(0)}\left(\Delta_{2}^{(0)}(\theta), 0, x_{0}\right)\right)\right\| d \theta \\
& +\varepsilon \int_{J_{\tau_{1}}^{+}, t} \| A\left(\Delta_{2}^{(0)}(\theta), x_{2}^{(0)}\left(\Delta_{2}^{(0)}(\theta), \tau_{1}, x_{1}^{+}\right)\right. \text {, } \\
& \left.x_{2}^{(0)}\left(\Delta_{2}^{(0)}\left(\Delta_{2}^{(0)}(\theta)\right), \tau_{1}, x_{1}^{+}\right), \dot{x}_{2}^{(0)}\left(\Delta_{2}^{(0)}\left(\Delta_{2}^{(0)}(\theta)\right), \tau_{1}, x_{1}^{+}\right)\right) \| \\
& \left.\cdot\left\|X\left(\Delta_{2}^{(0)}(\theta), x_{2}^{(0)}\left(\Delta_{2}^{(0)}(\theta), \tau_{1}, x_{1}^{+}\right)\right)\right\| d \theta\right\} \leq 2 \varepsilon^{2} K M^{2} \int_{\tau_{1}}^{t}(M \theta+1) d \theta \\
& +2 \varepsilon K M\left\|R_{1}^{(0)}\left(\tau_{1}, 0, x_{0}, \varepsilon\right)\right\| \int_{\tau_{1}}^{t} d \theta+\varepsilon \gamma(\varepsilon) \sqrt{n} K M \int_{J_{\tau_{1}}^{-}, t}\left|\Delta_{2}^{(0)}(\theta)\right| d \theta \\
& +\varepsilon^{2} K M \int_{J_{\tau_{1}, t}^{1}} d \theta \int_{0}^{\Delta_{2}^{(0)}(\theta)} \| A\left(l, x_{1}^{(0)}\left(l, 0, x_{0}\right), x_{1}^{(0)}\left(\Delta_{1}^{(0)}(l), 0, x_{0}\right),\right. \\
& \left.\dot{x}_{1}^{(0)}\left(\Delta_{1}^{(0)}(l), 0, x_{0}\right)\right)\|\cdot\| X\left(l, x_{1}^{(0)}\left(l, 0, x_{0}\right)\right) \| d l \\
& +\varepsilon^{2} K M^{2} \int_{J_{\tau_{1}, t}^{+}}\left(M \Delta_{2}^{(0)}(\theta)+1\right) d \theta \\
& +\varepsilon K M\left\|R_{1}^{(0)}\left(\tau_{1}, 0, x_{0}, \varepsilon\right)\right\| \int_{J_{\tau_{1}}^{+}, t} d \theta+\varepsilon \gamma(\varepsilon) K M \int_{\tau_{\tau_{1}}, t} d \theta \\
& +\varepsilon^{2} K M^{3}\left(\int_{J_{\tau_{1}, t}^{1}} d \theta+\int_{{J_{\tau_{1}}, t}^{+}} d \theta\right) \leq \varepsilon^{2} K M(M T+1)^{2}+\varepsilon \gamma(\varepsilon) \delta \sqrt{n} K M T \\
& +\varepsilon^{2} K M^{2} \int_{J_{\tau_{1}, t}^{1}} M \Delta_{2}^{(0)}(\theta) d \theta+\varepsilon^{2} K M^{2} \int_{J_{\tau_{1}, t}^{+}}\left(M \Delta_{2}^{(0)}(\theta)+1\right) d \theta \\
& +\varepsilon \omega_{1}^{(0)}\left(\varepsilon^{2}, T\right) K M \int_{J_{\tau_{1}}^{+}, t} d \theta+2 \varepsilon \omega_{1}^{(0)}\left(\varepsilon^{2}, T\right) K M T+\varepsilon \gamma(\varepsilon) K M \int_{\tau_{1}}^{t} d \theta
\end{aligned}
$$




$$
\begin{aligned}
& +\varepsilon^{2} K M^{3} \int_{\tau_{1}}^{t} d \theta \leq \varepsilon^{2} K M(M T+1)^{2}+\varepsilon \gamma(\hat{\sigma})(\hat{o} \sqrt{n}+1) K M T \\
& +\varepsilon^{2} K M^{2} \int_{\tau_{1}}^{t}(M \theta+1) d \theta+3 \varepsilon \omega_{1}^{(0)}\left(\varepsilon^{2}, T\right) K M T+\varepsilon^{2} K M^{3} T \\
& \leq 3 \varepsilon^{2} K M(M T+1)^{2} / 2+\varepsilon \gamma(\varepsilon)(\delta \sqrt{n}+1) K M T \\
& +3 \varepsilon \omega_{1}^{(0)}\left(\varepsilon^{2}, T\right) K M T+\varepsilon^{2} K M^{3} T \equiv \omega_{2}^{(0)}\left(\varepsilon^{2}, T\right),
\end{aligned}
$$

where

$$
\begin{aligned}
& J_{\tau_{1}, t}^{-} \cup J_{\tau_{1}, t}^{1} \cup J_{\tau_{1}, t}^{+}=\left(\tau_{1}, t\right], \\
& J_{\tau_{1}, t}^{-}=\left\{\theta: \theta \in\left(\tau_{1}, t\right] \wedge \Delta_{2}^{(0)}(\theta) \in[-\delta, 0]\right\}, \\
& J_{\tau_{1}, t}^{1}=\left\{\theta: \theta \in\left(\tau_{1}, t\right] \wedge \Delta_{2}^{(0)}(\theta) \in\left(0, \tau_{1}\right]\right\}, \\
& J_{\tau_{1}, t}^{+}=\left(\tau_{1}, t\right] \backslash\left(J_{\tau_{1}, t}^{-} \cup J_{\tau_{1}, t}^{1}\right) .
\end{aligned}
$$

Therefore, the function $\widetilde{x}_{2}^{(0)}\left(t, \tau_{1}, x_{1}^{+}\right)$approximates the solution $x_{2}^{(0)}\left(t, \tau_{1}, x_{1}^{+}\right)$of the system $(13)$ on the interval $\left(\tau_{1}, t\right] \subset(0, T]$ to a precision of order $\varepsilon^{2}$.

It can be shown that after the moment $\tau_{1}$ the trajectory $(t, x(t))$ does not again meet the hypersurface $\sigma_{1}$.

Indeed, solving the equation

$$
t=t_{1}\left(x_{2}^{(0)}\left(t, \tau_{1}, x_{1}^{+}\right)\right),
$$

we obtain its root

$$
\bar{t}_{1}=\tau_{1}+\varepsilon \frac{\partial t_{1}\left(x_{0}\right)}{\partial x} I_{1}^{(0)}+O\left(\varepsilon^{2}\right)
$$

Whence, and from the condition $6^{\circ}$ of Theorem 1 and the continuity of the vector-function $I_{1}(x)$ it follows that the inequality $\bar{t}_{1}<\tau_{1}$ is fulfilled for sufficiently small values of $\varepsilon$. Thus, we showed that the trajectory ( $t$, $x(t))$ for $t>\tau_{1}$ does not again meet the hypersurface $\sigma_{1}$.

The moment at which the trajectory $(t, x(t))$ meets the hypersurface $\sigma_{2}$ is

$$
\tau_{2}=t_{2}^{(0)}+\varepsilon \Theta_{2}^{(0)}+O\left(\varepsilon^{2}\right),
$$

where

$$
\Theta_{2}^{(0)}=\frac{\partial t_{2}\left(x_{0}\right)}{\partial x}\left[\int_{0}^{t_{2}^{(0)}} A\left(\theta, x_{0}, x_{0}, 0\right) X\left(\theta, x_{0}\right) d \theta+I_{1}^{(0)}\right]
$$


$t_{2}^{(0)}>t_{1}^{(0)}$ implies that $\tau_{2}>\tau_{1}$ when $\varepsilon$ is sufficiently small.

Thus

$$
\begin{aligned}
x(t) & =x_{2}^{(0)}\left(t, \tau_{1}, x_{1}^{+}\right)=\widetilde{x}_{2}^{(0)}\left(t, \tau_{1}, x_{1}^{+}\right)+R_{2}^{(0)}\left(t, \tau_{1}, x_{1}^{+}, \varepsilon\right) \\
& =\widetilde{x}_{1}^{(0)}\left(t, 0, x_{0}\right)+\varepsilon I_{1}^{(0)}+R_{1}^{(0)}\left(\tau_{1}, 0, x_{0}, \varepsilon\right)+R_{2}^{(0)}\left(t, \tau_{1}, x_{1}^{+}, \varepsilon\right)
\end{aligned}
$$

for $\tau_{1}<t \leq \tau_{2}=t_{2}^{(0)}+\varepsilon \Theta_{2}^{(0)}+O\left(\varepsilon^{2}\right)$ and

$$
x_{2}^{+}=x_{2}^{(0)}\left(\tau_{2}, \tau_{1}, x_{1}^{+}\right)+\varepsilon I_{2}\left(x_{2}^{(0)}\left(\tau_{2}, \tau_{1}, x_{1}^{+}\right)\right),
$$

i.e.

$$
\begin{aligned}
x_{2}^{+}= & \widetilde{x}_{1}^{(0)}\left(\tau_{2}, 0, x_{0}\right)+\varepsilon\left(I_{1}^{(0)}+I_{2}^{(0)}\right)+R_{1}^{(0)}\left(\tau_{1}, 0, x_{0}, \varepsilon\right) \\
& +R_{2}^{(0)}\left(\tau_{2}, \tau_{1}, x_{1}^{+}, \varepsilon\right)=x_{0}+\varepsilon \int_{0}^{\tau_{2}} A\left(\theta, x_{0}, x_{0}, 0\right) X\left(\theta, x_{0}\right) d \theta \\
& +\varepsilon\left(I_{1}^{(0)}+I_{2}^{(0)}\right)+R_{1}^{(0)}\left(\tau_{1}, 0, x_{0}, \varepsilon\right)+R_{2}^{(0)}\left(\tau_{2}, \tau_{1}, x_{1}^{+}, \varepsilon\right),
\end{aligned}
$$

where $I_{2}^{(0)} \equiv I_{2}\left(x_{2}^{(0)}\left(\tau_{2}, \tau_{1}, x_{1}^{+}\right)\right)$.

In the general case $\left(s=\overline{\left.2,\left(d_{1}+1\right)\right)}\right.$ we denote by $x_{s}^{(0)}\left(t, \tau_{s-1}, x_{s-1}^{+}\right)$ the solution of the system

$$
\begin{aligned}
& x_{s}^{(0)}\left(t, \tau_{s-1}, x_{s-1}^{+}\right) \\
& =\left\{\begin{array}{l}
x_{s-1}^{+}+\varepsilon \int_{\tau_{s-1}}^{t} A\left(\theta, x_{s}^{(0)}\left(\theta, \tau_{s-1}, x_{s-1}^{+}\right), x_{s}^{(0)}\left(\Delta_{s}^{(0)}(\theta), \tau_{s-1}, x_{s-1}^{+}\right),\right. \\
\left.\dot{x}_{s}^{(0)}\left(\Delta_{s}^{(0)}(\theta), \tau_{s-1}, x_{s-1}^{+}\right)\right) X\left(\theta, x_{s}^{(0)}\left(\theta, \tau_{s-1}, x_{s-1}^{+}\right)\right) d \theta, \quad t>\tau_{s-1} \\
x_{s-1}^{(0)}\left(t, \tau_{s-2}, x_{s-2}^{+}\right), \quad-\delta \leq t \leq \tau_{s-1},
\end{array}\right. \\
& \dot{x}_{s}^{(0)}\left(t, \tau_{s-1}, x_{s-1}^{+}\right)=\dot{x}_{s-1}^{(0)}\left(t, \tau_{s-2}, x_{s-2}^{+}\right), \quad-\delta \leq t \leq \tau_{s-1},
\end{aligned}
$$

where $\Delta_{s}^{(0)}(t)=\Delta\left(t, x_{s}^{(0)}\left(t, \tau_{s-1}, x_{s-1}^{+}\right)\right)$,

$$
\begin{aligned}
x_{s-1}^{+}= & x_{s-1}^{(0)}\left(\tau_{s-1}, \tau_{s-2}, x_{s-2}^{+}\right)+\varepsilon I_{s-1}\left(x_{s-1}^{(0)}\left(\tau_{s-1}, \tau_{s-2}, x_{s-2}^{+}\right)\right) \\
= & x_{0}+\varepsilon \int_{0}^{\tau_{s-1}} A\left(\theta, x_{0}, x_{0}, 0\right) X\left(\theta, x_{0}\right) d \theta+\varepsilon \sum_{i=1}^{s-1} I_{i}^{(0)} \\
& +\sum_{i=1}^{s-1} R_{i}^{(0)}\left(\tau_{i}, \tau_{i-1}, x_{i-1}^{+}, \varepsilon\right), \\
I_{s-1}^{(0)} \equiv & I_{s-1}\left(x_{s-1}^{(0)}\left(\tau_{s-1}, \tau_{s-2}, x_{s-2}^{+}\right)\right), \quad x_{0}^{+}=x_{0} .
\end{aligned}
$$

The solution of (14) coincides with the solution of the system of functional-differential equations (1) with impulses on the interval $[-\delta$, 
$\tau_{s}$, where $\tau_{s}$ is the moment it which the trijectory $(t . x(t))$ meets the hypersurface $\sigma_{\text {. }}$.

If

$$
\widetilde{x}_{s}^{(0)}\left(t, \tau_{s-1}, x_{s-1}^{+}\right)=x_{s-1}^{(0)}+\varepsilon \int_{\tau_{s-1}}^{t} A\left(\theta, x_{0}, x_{0}, 0\right) X\left(\theta, x_{0}\right) d \theta
$$

it can be shown, as we did in the cases $s=1$ and $s=2$ that the difference

$$
R_{s}^{(0)}\left(t, \tau_{s-1}, x_{s-1}^{+}, \varepsilon\right)=x_{s}^{(0)}\left(t, \tau_{s-1}, x_{s-1}^{+}\right)-\widetilde{x}_{s}^{(0)}\left(t, \tau_{s-1}, x_{s-1}^{+}\right)
$$

on the interval $0<\tau_{s-1}<t \leq T$ satisfies the inequality

$$
\begin{aligned}
& \left\|R_{s}^{(0)}\left(t, \tau_{s-1}, x_{s-1}^{+}, \varepsilon\right)\right\| \leq 3 \varepsilon^{2} K M(M T+s-1)^{2} / 2 \\
& \quad+\varepsilon \gamma(\varepsilon)(\delta \sqrt{n}+1) K M T+3 \varepsilon \sum_{i=1}^{s-1} \omega_{i}^{(0)}\left(\varepsilon^{2}, T\right) K M T+\varepsilon^{2} K M^{3} T \\
& \equiv \omega_{s}^{(0)}\left(\varepsilon^{2}, T\right) .
\end{aligned}
$$

Therefore, the function $\widetilde{x}_{s}^{(0)}\left(t, \tau_{s-1}, x_{s-1}^{+}\right)$approximates the solution $x(t)$ of the system of functional-differential equations (1) with impulses on the interval $\left(\tau_{s-1}, t\right] \subset(0, T]$ to a precision of order $\varepsilon^{2}$, etc.

Since for $s=\overline{2,\left(d_{1}+1\right)}$ we have

$$
\begin{aligned}
\widetilde{x}_{s}^{(0)}\left(t, \tau_{s-1}, x_{s-1}^{+}\right)=x_{s-1}^{+}+\varepsilon \int_{\tau_{s-1}}^{\iota} A\left(\theta, x_{0}, x_{0}, 0\right) X\left(\theta, x_{0}\right) d \theta \\
=x_{0}+\varepsilon \int_{0}^{\tau_{s-1}} A\left(\theta, x_{0}, x_{0}, 0\right) X\left(\theta, x_{0}\right) d \theta+\varepsilon \sum_{i=1}^{s-1} I_{i}^{(0)} \\
\quad+\sum_{i=1}^{s-1} R_{i}^{(0)}\left(\tau_{i}, \tau_{i-1}, x_{i-1}^{+}, \varepsilon\right)+\varepsilon \int_{\tau_{s-1}}^{t} A\left(\theta, x_{0}, x_{0}, 0\right) X\left(\theta, x_{0}\right) d \theta \\
=\widetilde{x}_{1}^{(0)}\left(t, 0, x_{0}\right)+\varepsilon \sum_{i=1}^{s-1} I_{i}^{(0)}+\sum_{i=1}^{s-1} R_{i}^{(0)}\left(\tau_{i}, \tau_{i-1}, x_{i-1}^{+}, \varepsilon\right),
\end{aligned}
$$

then

$$
\begin{aligned}
x(t)= & x_{s}^{(0)}\left(t, \tau_{s-1}, x_{s-1}^{+}\right)=\widetilde{x}_{1}^{(0)}\left(t, 0, x_{0}\right)+\varepsilon \sum_{i=0}^{s-1} I_{i}^{(0)} \\
& +\sum_{i=0}^{s-1} R_{i}^{(0)}\left(\tau_{i}, \tau_{i-1}, x_{s-1}^{+}, \varepsilon\right)+R_{3}^{(0)}\left(t, \tau_{s-1}, x_{s-1}^{+}, \varepsilon\right)
\end{aligned}
$$

for

$$
t_{s-1}^{(0)}+\varepsilon \Theta_{s-1}^{(0)}+\gamma_{s-1} O\left(\varepsilon^{2}\right)=\tau_{s-1}<t \leq \tau_{s}=t_{s}^{(0)}+\varepsilon \Theta_{s}^{(0)}+O\left(\varepsilon^{2}\right),
$$

where 


$$
\begin{aligned}
& \Theta_{s}^{(0)}=\frac{\partial t_{s}\left(x_{0}\right)}{\partial x}\left[\int_{0}^{t_{s}^{(0)}} A\left(\theta, x_{0}, x_{0}, 0\right) X\left(\theta, x_{0}\right) d \theta+\sum_{i=1}^{s-1} I_{i}^{(0)}\right], \\
& t_{0}^{(0)}=\Theta_{0}^{(0)}=\gamma_{0}=0, I_{0}^{(0)}=R_{0}^{(0)}\left(\tau_{0}, \tau_{-1}, x_{-1}^{+}, \varepsilon\right)=0, \gamma_{s}=1, s=\overline{1, d_{1}},
\end{aligned}
$$

as well as for

$$
t_{d_{1}}^{(0)}+\varepsilon \Theta_{d_{1}}^{(0)}+O\left(\varepsilon^{2}\right)=\tau_{d_{1}}<t \leq T, \quad s=d_{1}+1 .
$$

Therefore

$$
\begin{aligned}
x(T)= & x_{d_{1}+1}^{(0)}\left(T, \tau_{d_{1}}, x_{d_{1}}^{+}\right)=x_{0}+\varepsilon \int_{0}^{t} A\left(\theta, x_{0}, x_{0}, 0\right) X\left(\theta, x_{0}\right) d \theta \\
& +\varepsilon \sum_{i=0}^{d_{1}} I_{i}^{(0)}+\sum_{i=0}^{d_{1}} R_{i}^{(0)}\left(\tau_{i}, \tau_{i-1}, x_{i-1}^{+}, \varepsilon\right)+R_{d_{1}+1}^{(0)}\left(T, \tau_{d_{1}}, x_{d_{1}}^{+}, \varepsilon\right)
\end{aligned}
$$

Let $\bar{x}(t)$ be the solution of the averaged system (5) with initial condition (6). Then for $t \geq 0$

$$
\bar{x}(t)=x_{0}+\varepsilon \int_{0}^{t}\left[A_{0}(\bar{x}(\theta)) X(\theta, \bar{x}(\theta))+I_{0}(\bar{x}(\theta))\right] d \theta
$$

and

$$
\bar{x}(T)=x_{0}+\varepsilon \int_{0}^{T}\left[A_{0}(\bar{x}(\theta)) X(\theta, \bar{x}(\theta))+I_{0}(\bar{x}(\theta))\right] d \theta .
$$

We shall estimate the difference $x(T)-\bar{x}(T)$. For the purpose, taking into account (8), we write down $x(T)$ in the form

$$
\begin{aligned}
x(T)= & x_{0}+\varepsilon I_{0}\left(x_{0}\right) T+\varepsilon A_{0}\left(x_{0}\right) \int_{0}^{T} X\left(\theta, x_{0}\right) d \theta \\
& +\varepsilon \int_{0}^{T}\left[A\left(\theta, x_{0}, x_{0}, 0\right)-A_{0}\left(x_{0}\right)\right] X\left(\theta, x_{0}\right) d \theta \\
& +\varepsilon\left[\sum_{i=0}^{d_{1}} I_{i}^{(0)}-I_{0}\left(x_{0}\right) T\right]+\sum_{i=0}^{d_{1}} R_{i}^{(0)}\left(\tau_{i}, \tau_{i-1}, x_{i-1}^{+}, \varepsilon\right) \\
& +R_{d_{1}+1}^{(0)}\left(T, \tau_{d_{1}}, x_{d_{1}}^{+}, \varepsilon\right) .
\end{aligned}
$$

For each $x \in D$ we define the operator $B_{p}(p=1,2, \cdots)$ in the following way

$$
B_{p} x=x+\varepsilon I_{0}(x) T+\varepsilon A_{0}(x) \int_{(p-1) T}^{p T} X(\theta, x) d \theta .
$$

From (16), in virtue of (8), the conditions of Theorem 1 , the generalized theorem for the mean values in Integral Calculus, and the Cauchy 
inequality in the discrete case we obtain

(17)

$$
\begin{aligned}
& \left\|x(T)-B_{1} x_{0}\right\| \leq \varepsilon\left\|\int_{0}^{T}\left[A\left(\theta, x_{0}, x_{0}, 0\right)-A_{0}\left(x_{0}\right)\right] X\left(\theta, x_{0}\right) d \theta\right\| \\
& +\varepsilon\left\|\sum_{i=0}^{d_{1}} I_{i}^{(0)}-I_{0}\left(x_{0}\right) T\right\|+\sum_{i=0}^{d_{1}+1} \omega_{i}^{(0)}\left(\varepsilon^{2}, T\right) \\
& \leq \varepsilon \alpha(T) T / 2+\varepsilon\left\|\sum_{i=1}^{d_{1}} I_{i}\left(x_{0}\right)-I_{0}\left(x_{0}\right) T\right\|+\varepsilon\left\|\sum_{i=1}^{d_{1}}\left(I_{i}^{(0)}-I_{i}\left(x_{0}\right)\right)\right\| \\
& +\sum_{i=0}^{d_{1}+1} \omega_{i}^{(0)}\left(\varepsilon^{2}, T\right) \\
& \leq \varepsilon \alpha(T) T+\varepsilon \sum_{i=1}^{d_{1}} \| I_{i}\left(x_{i}^{(0)}\left(\tau_{i}, \tau_{i-1}, x_{i-1}^{+}\right)-I_{i}\left(x_{0}\right) \|+\sum_{i=0}^{d_{1}+1} \omega_{i}^{(0)}\left(\varepsilon^{2}, T\right)\right. \\
& \leq \varepsilon \alpha(T) T+\varepsilon K \sum_{i=1}^{d_{1}}\left\|x_{i}^{(0)}\left(\tau_{i}, \tau_{i-1}, x_{i-1}^{+}\right)-x_{0}\right\|+\sum_{i=0}^{d_{1}+1} \omega_{i}^{(0)}\left(\varepsilon^{2}, T\right) \\
& =\varepsilon \alpha(T) T+\varepsilon K \sum_{i=1}^{d_{1}} \| x_{0}+\varepsilon \int_{0}^{\tau_{i}} A\left(\theta, x_{0}, x_{0}, 0\right) X\left(\theta, x_{0}\right) d \theta \\
& +\varepsilon \sum_{l=0}^{i-1} I_{l}^{(0)}+\sum_{l=0}^{i} R_{l}^{(0)}\left(\tau_{l}, \tau_{l-1}, x_{l-1}^{+}, \varepsilon\right)-x_{0} \|+\sum_{i=0}^{d_{1}+1} \omega_{i}^{(0)}\left(\varepsilon^{2}, T\right) \\
& \leq \varepsilon \alpha(T) T+\varepsilon^{2} K M^{2} T d_{1}+\varepsilon^{2} K \sum_{i=1}^{d_{1}} \sum_{l=0}^{i-1}\left\|I_{l}^{(0)}\right\| \\
& +\varepsilon K \sum_{i=1}^{d_{1}} \sum_{l=0}^{i}\left\|R_{l}^{(0)}\left(\tau_{l}, \tau_{l-1}, x_{l-1}^{+}, \hat{\varepsilon}\right)\right\|+\sum_{i=0}^{a_{1}+1} \omega_{i}^{(0)}\left(\varepsilon^{2}, T\right) \\
& \leq \varepsilon \alpha(T) T+\varepsilon^{2} K M d_{1}\left(2 M T+d_{1}-1\right) / 2+\sum_{i=0}^{d_{1}+1} \omega_{i}^{(0)}\left(\varepsilon^{2}, T\right) \\
& +\varepsilon K \sum_{i=1}^{d_{1}} \sum_{l=0}^{i} \omega_{l}^{(0)}\left(\varepsilon^{2}, T\right) \leq \varepsilon \alpha(T) T+\varepsilon^{2} M_{1},
\end{aligned}
$$

where $\omega_{0}^{(0)}\left(\varepsilon^{2}, T\right) \equiv 0, M_{1}=M_{1}\left(T, d_{1}\right)$ is a constant. For $t \geq 0, \tau \in[0, T)$ and $x \in D$ we have

$$
\begin{aligned}
& \left\|A_{0}(x)\right\|=\left\|\lim _{T \rightarrow \infty} \frac{1}{T} \int_{t}^{t+T} A(\theta, x, x, 0) d \theta\right\| \leq M ; \\
& \left\|I_{0}(x)\right\| \leq \lim _{T \rightarrow \infty} \frac{1}{T} \sum_{t<t_{i}<t+T}\left\|I_{i}(x)\right\| \leq M d ; \\
& \left\|\bar{x}(\tau)-x_{0}\right\| \leq \varepsilon M(M+d) T ;
\end{aligned}
$$




$$
\begin{gathered}
\left\|A_{0}(\bar{x}(\tau))-A_{0}\left(x_{0}\right)\right\| \leq \lim _{T \rightarrow \infty} \frac{1}{T} \| \int_{t}^{t+T}[A(\theta, \bar{x}(\tau), \bar{x}(\tau), 0) \\
\left.-A\left(\theta, x_{0}, x_{0}, 0\right)\right] d \theta \| \leq 2 \varepsilon(M+d) K M T ; \\
\left\|X(\tau, \bar{x}(\tau))-X\left(\tau, x_{0}\right)\right\| \leq \varepsilon(M+d) K M T ; \\
\left\|I_{0}(\bar{x}(\tau))-I_{0}\left(x_{0}\right)\right\| \leq \lim _{T \rightarrow \infty} \frac{1}{T}\left\|\sum_{t<t_{i}<t+T} I_{i}(\bar{x}(\tau))-I_{i}\left(x_{0}\right)\right\| \\
\leq \varepsilon d(M+d) K M T .
\end{gathered}
$$

Making use of these estimates, we get

$$
\begin{aligned}
& \left\|\bar{x}(T)-B_{1} x_{0}\right\|=\| x_{0}+\varepsilon \int_{0}^{T}\left[A_{0}(\bar{x}(\theta)) X(\theta, \bar{x}(\theta))+I_{0}(\bar{x}(\theta))\right] d \theta \\
& \quad-x_{0}-\varepsilon I_{0}\left(x_{0}\right) T-\varepsilon A_{0}\left(x_{0}\right) \int_{0}^{T} X\left(\theta, x_{0}\right) d \theta \| \\
& \leq \varepsilon \int_{0}^{T}\left\{\left\|A_{0}(\bar{x}(\theta))-A_{0}\left(x_{0}\right)\right\| \cdot\|X(\theta, \bar{x}(\theta))\|\right. \\
& \quad+\left\|A_{0}\left(x_{0}\right)\right\| \cdot\left\|X(\theta, \bar{x}(\theta))-X\left(\theta, x_{0}\right)\right\| \\
& \left.\quad+\left\|I_{0}(\bar{x}(\theta))-I_{0}\left(x_{0}\right)\right\|\right\} d \theta \leq \varepsilon^{2}(M+d)(3 M+d) K M T^{2} .
\end{aligned}
$$

(17) and (18) yield the inequality

$$
\begin{aligned}
\|x(T)-\bar{x}(T)\| & \leq\left\|x(T)-B_{1} x_{0}\right\|+\left\|\bar{x}(T)-B_{1} x_{0}\right\| \\
& \leq \varepsilon \alpha(T) T+\varepsilon^{2} \bar{M}
\end{aligned}
$$

where $\bar{M}=(M+d)(3 M+d) K M T^{2}+M_{1}$.

Thus we obtained an estimate for $\|x(T)-\bar{x}(T)\|$ and established the proximity of the points $x(T)$ and $\bar{x}(T)$.

Since $\bar{x}(T)$ belongs to the domain $D$ with its neighbourhood of radius $\rho$, then (18) and (19) imply that the points $B_{1} x_{0}$ and $x(T)$ also belong to the domain $D$.

Thus inequality (7) is substantiated for $p=1$.

We introduce the notations

$$
\begin{aligned}
& \tau_{i}^{(r-1)} \equiv \tau_{d_{0}+d_{1}+\cdots+d_{s-1}+i}, \\
& x_{i}^{(r-1)+} \equiv x_{d_{0}+d_{1}+\cdots+d_{r-1}+i}^{+}, \quad d_{0}=0, \quad i=\overline{1, d_{r}} \\
& \tau_{0}^{(r-1)} \equiv(r-1) T, \quad \tau_{d_{r}+1}^{(r-1)} \equiv r T,
\end{aligned}
$$




$$
x_{0}^{(r-1)+} \equiv x((r-1) T), \quad x_{d_{r}+1}^{(r-1)+} \equiv x(r T), \quad r=1,2, \cdots .
$$

Note that with the notations thus introduced we have

$$
\tau_{0}^{(r-1)} \equiv \tau_{a_{r-1}+1}^{(r-2)} \quad \text { and } \quad x_{0}^{(r-1)+} \equiv x_{d_{r-1}+1}^{(r-2)+}, \quad r=2,3, \cdots
$$

Let us assume that for $p=r, r \geq 2$ inequality (7) is fulfilled and we have results of the type of (15) and (17)-(19), i.e. we have

$$
\begin{aligned}
x(t)= & x_{s}^{(r-1)}\left(t, \tau_{s-1}^{(r-1)}, x_{s-1}^{(r-1)+}\right)=\widetilde{x}_{1}^{(r-1)}(t,(r-1) T, x((r-1) T)) \\
& +\varepsilon \sum_{i=0}^{s-1} I_{i}^{(r-1)}+\sum_{i=0}^{s-1} R_{i}^{(r-1)}\left(\tau_{i}^{(r-1)}, \tau_{i-1}^{(r-1)}, x_{i-1}^{(r-1)+}, \varepsilon\right) \\
& +R_{s}^{(r-1)}\left(t, \tau_{s-1}^{(r-1)} x_{s-1}^{(r-1)+}, \varepsilon\right)
\end{aligned}
$$

for

$$
t_{s-1}^{(r-1)}+\varepsilon \Theta_{s-1}^{(r-1)}+\gamma_{s-1} O\left(\varepsilon^{2}\right)=\tau_{s-1}^{(r-1)}<t \leq \tau_{s}^{(r-1)}=t_{s}^{(r-1)}+\varepsilon \Theta_{s}^{(r-1)}+O\left(\varepsilon^{2}\right),
$$

where

$$
\begin{aligned}
\Theta_{s}^{(r-1)}= & \frac{\partial t_{d_{1}+\cdots+d_{r-1}+s}(x((r-1) T))}{\partial x} \\
& \cdot\left[\int_{(r-1) T}^{t_{s}^{(r-1)}} A(\theta, x((r-1) T), x((r-1) T), 0)\right. \\
& \left.\cdot X(\theta, x((r-1) T)) d \theta+\sum_{i=0}^{s-1} I_{i}^{(r-1)}\right], \\
t_{0}^{(r-1)}= & r T, \quad \Theta_{0}^{(r-1)}=\gamma_{0}=0, \\
I_{0}^{(r-1)}= & 0, \quad R_{0}^{(r-1)}\left(\tau_{0}^{(r-1)}, \tau_{-1}^{(r-1)}, x_{-1}^{(r-1)+}, \varepsilon\right)=0, \quad \gamma_{s}=1, \quad s=\overline{1, d_{r}}
\end{aligned}
$$

as well as for

$$
\begin{aligned}
& t_{d_{r}}^{(r-1)}+\varepsilon \Theta_{d_{r}}^{(r-1)}+O\left(\varepsilon^{2}\right)=\tau_{d_{r}}^{(r-1)}<t \leq \tau_{d_{r}+1}^{(r-1)}=r T, \quad s=d_{r}+1 ; \\
& \left\|x(r T)-B_{r} x((r-1) T)\right\| \leq \varepsilon \alpha(T) T+\varepsilon^{2} K M d_{r}\left(2 M T+d_{r}-1\right) / 2 \\
& \quad+\sum_{i=0}^{d_{r}+1} \omega_{i}^{(r-1)}\left(\varepsilon^{2}, T\right)+\varepsilon K \sum_{i=1}^{d_{r}} \sum_{l=0}^{i} \omega_{l}^{(r-1)}\left(\varepsilon^{2}, T\right) \\
& =\varepsilon \alpha(T) T+\varepsilon^{2} M_{r},
\end{aligned}
$$

where $\omega_{0}^{(r-1)}\left(\varepsilon^{2}, T\right) \equiv 0, M_{r}=M_{r}\left(T, d_{1}, \cdots, d_{r}\right)$ is a constant;

$$
\begin{aligned}
& \left\|B_{r} x((r-1) T)-B_{r} \bar{x}((r-1) T)\right\| \\
& \quad \leq[1+\varepsilon(3 M+d) K T] \sum_{i=0}^{r-2}[1+\varepsilon(3 M+d) K T]^{i}\left[\varepsilon \alpha(T) T+\varepsilon^{2} \bar{M}\right],
\end{aligned}
$$




$$
\begin{aligned}
& \text { where } \bar{M}=(M+d)(3 M+d) K M T^{2}+\max _{i=1,(r-1)} M_{i} ; \\
& \qquad B_{r} \bar{x}((r-1) T)-\bar{x}(r T) \| \leq \varepsilon^{2}(M+d)(3 M+d) K M T^{2} ; \\
& \|x(r T)-\bar{x}(r T)\| \leq\left\|x(r T)-B_{r} x((r-1) T)\right\| \\
& \quad+\left\|B_{r} x((r-1) T)-B_{r} \bar{x}((r-1) T)\right\|+\left\|B_{r} \bar{x}((r-1) T)-\bar{x}(r T)\right\| \\
& \leqq \\
& \sum_{i=0}^{r-1}[1+\varepsilon(3 M+d) K T]^{i}\left[\varepsilon \alpha(T) T+\varepsilon^{2} \bar{M}\right],
\end{aligned}
$$

where $\bar{M}=(M+d)(3 M+d) K M T^{2}+\max _{i=\overline{1, r}} M_{i}$.

Let $d_{r+1}$ points lie on the interval $(r T,(r+1) T)$

$$
t_{d_{1}+\cdots+d_{r+1}}(\bar{x}(r T)), \cdots, t_{d_{1}+\cdots+d_{r}+d_{r+1}}(\bar{x}(r T)),
$$

in which case

$$
t_{d_{1}+\cdots+d_{r}+i}(\bar{x}(r T))<t_{d_{1}+\cdots+d_{r}+i+1}(\bar{x}(r T)), \quad i=\overline{1,\left(d_{r+1}-1\right)}
$$

Then from (7) for $p=r$ and from the continuity of the functions $t_{i}(x), i=1,2, \cdots$ it follows that if $\varepsilon$ is sufficiently small, $d_{r+1}$ points lie on the interval $(r T,(r+1) T)$

$$
\begin{aligned}
& t_{d_{1}+\cdots+d_{r+1}}(x(r T))=t_{1}^{(r)}, \cdots, \\
& t_{d_{1}+\cdots+d_{r}+d_{r+1}}(x(r T))=t_{d_{r+1}}^{(r)},
\end{aligned}
$$

where

$$
t_{i}^{(r)}<t_{i+1}^{(r)}, \quad i=\overline{1,\left(d_{r+1}-1\right)} .
$$

The conditions of Lemma 1 and (7) for $p=r$ imply that if $\varepsilon$ is sufficiently small there exists a constant $\beta_{r} \in[-\beta, 0)$ such that for $i=$ $\overline{1, d_{r+1}}$ the inequality

$$
\frac{\partial t_{d_{1}+\cdots+d_{r+i}}(x(r T))}{\partial x} I_{a_{1}+\cdots+d_{r}+i}(x(r T)) \leq \beta_{r}<0
$$

holds.

We shall prove the validity of (7) for $p=r+1$.

The solution of the system of functional-differential equations (1) with impulses, which we accept to be constructed on the intervals $((p-1) T, p T], p=\overline{1, r}$ will be continued onto the next interval ( $r^{T} T$, $(r+1) T]$, denoting for the sake of brevity $x(p T)$ by $x_{p T}$. 
Let $x_{1}^{(r)}\left(t, r T, x_{r T}\right)$ be a solution of the system

$$
\begin{aligned}
& x_{1}^{(r)}\left(t, r T, x_{r T}\right) \\
& =\left\{\begin{array}{l}
x_{r T}+\varepsilon \int_{r T}^{t} A\left(\theta, x_{1}^{(r)}\left(\theta, r T, x_{r T}\right), x_{1}^{(r)}\left(\Delta_{1}^{(r)}(\theta), r T, x_{r T}\right)\right), \\
\left.\quad \dot{x}_{1}^{(r)}\left(\Delta_{1}^{(r)}(\theta), r T, x_{r T}\right)\right) X\left(\theta, x_{1}^{(r)}\left(\theta, r T, x_{r T}\right)\right) d \theta, \quad t>r T, \\
x_{d_{r}+1}^{(r-1)}\left(t, \tau_{d_{r}}^{(r-1)}, x_{d_{r}}^{(r-1)+}\right), \quad-\delta \leq t \leq r T,
\end{array}\right. \\
& \dot{x}_{1}^{(r)}\left(t, r T, x_{r T}\right)=\dot{x}_{d_{r}+1}^{(r-1)}\left(t, \tau_{d_{r}}^{(r-1)}, x_{d_{r}}^{(r-1)+}\right), \quad-\delta \leq t \leq r T,
\end{aligned}
$$

where

$$
\Delta_{1}^{(r)}(t)=\Delta\left(t, x_{1}^{(r)}\left(t, r T, x_{r T}\right)\right) .
$$

The solution of (22) coincides with the solution of the system of functional-differential equations (1) with impulses until the moment $\tau_{1}^{(r)}$ at which the trajectory $(t, x(t))$ meets the hypersurface $\sigma_{d_{1}+\cdots+d_{r}+1}$, i.e. for

$$
t \in\left[-\delta, \tau_{1}^{(r)}\right], \quad x(t)=x_{1}^{(r)}\left(t, r T, x_{r T}\right) .
$$

We consider the function

$$
\widetilde{x}_{1}^{(r)}\left(t, r T, x_{r T}\right)=x_{r T}+\varepsilon \int_{r T}^{t} A\left(\theta, x_{r T}, x_{r T}, 0\right) X\left(\theta, x_{r T}\right) d \theta .
$$

For $r T<t \leq(r+1) T$ we have

$$
\begin{aligned}
& \left\|R_{1}^{(r)}\left(t, r T, x_{r T}, \varepsilon\right)\right\|=\left\|x_{1}^{(r)}\left(t, r T, x_{r T}\right)-\widetilde{x}_{1}^{(r)}\left(t, r T, x_{r T}\right)\right\| \\
& \leq \varepsilon \int_{r T}^{t} \| A\left(\theta, x_{1}^{(r)}\left(\theta, r T, x_{r T}\right), x_{1}^{(r)}\left(\Delta_{1}^{(r)}(\theta), r T, x_{r T}\right),\right. \\
& \left.\dot{x}_{1}^{(r)}\left(\Delta_{1}^{(r)}(\theta), r T, x_{r T}\right)\right) X\left(\theta, x_{1}^{(r)}\left(\theta, r T, x_{r T}\right)\right) \\
& \quad-A\left(\theta, x_{r T}, x_{r T}, 0\right) X\left(\theta, x_{r T}\right) \| d \theta \\
& \leq \varepsilon \int_{r T}^{t}\left\{\| A\left(\theta, x_{1}^{(r)}\left(\theta, r T, x_{r T}\right), x_{1}^{(r)}\left(\Delta_{1}^{(r)}(\theta), r T, x_{r T}\right),\right.\right. \\
& \left.\quad \dot{x}_{1}^{(r)}\left(\Delta_{1}^{(r)}(\theta), r T, x_{r T}\right)\right)-A\left(\theta, x_{r T}, x_{r T}, 0\right) \| \\
& \cdot\left\|X\left(\theta, x_{1}^{(r)}\left(\theta, r T, x_{r T}\right)\right)\right\|+\left\|A\left(\theta, x_{r T}, x_{r T}, 0\right)\right\| \\
& \left.\cdot\left\|X\left(\theta, x_{1}^{(r)}\left(\theta, r T, x_{r T}\right)\right)-X\left(\theta, x_{r T}\right)\right\|\right\} d \theta \\
& \leq \varepsilon K M \int_{r T}^{t}\left\{2\left\|x_{1}^{(r)}\left(0, r T, x_{r T}\right)-x_{r T}\right\|+\| x_{1}^{(r)}\left(\Delta_{1}^{(r)}(\theta), r T, x_{r T}\right)\right.
\end{aligned}
$$




$$
\begin{aligned}
& \left.-x_{r T}\|+\| \dot{x}_{1}^{(r)}\left(\Delta_{1}^{(r)}(\theta), r T, x_{r T}\right) \|\right\} d \theta \\
& \leq 2 \varepsilon^{2} K M \int_{r T}^{t} d \theta \int_{r T}^{\theta} \| A\left(l, x_{1}^{(r)}\left(l, r T, x_{r_{T}}\right), x_{1}^{(r)}\left(\Delta_{1}^{(r)}(l), r T, x_{r T}\right)\right. \\
& \left.\dot{x}_{1}^{(r)}\left(\Delta_{1}^{(r)}(l), r T, x_{r T}\right)\right)\|\cdot\| X\left(l, x_{1}^{(r)}\left(l, r T, x_{r T}\right)\right) \| d l \\
& +\varepsilon K M\left\{\sum _ { i = 1 } ^ { d _ { r + 1 } } \int _ { J _ { r T , t } ^ { i } } \left[\left\|x_{i}^{(r-1)}\left(\Delta_{1}^{(r)}(\theta), \tau_{i-1}^{(r-1)}, x_{i-1}^{(r-1)+}\right)-x_{(r-1) T}\right\|\right.\right. \\
& \left.+\left\|x_{r T}-x_{(r-1) T}\right\|\right] d \theta+\varepsilon \int_{\tilde{J}_{T T, t}^{+}} d \theta \int_{r T}^{\Delta_{1}^{(r)}(\theta)} \| A\left(l, x_{1}^{(r)}\left(l, r T, x_{r T}\right),\right. \\
& \left.x_{1}^{(r)}\left(\Delta_{1}^{(r)}(l), r T, x_{r T}\right), \dot{x}_{1}^{(r)}\left(\Delta_{1}^{(r)}(l), r T, x_{r T}\right)\right) \| \\
& \left.\cdot\left\|X\left(l, x_{1}^{(r)}\left(l, r T, x_{r T}\right)\right)\right\| d l\right\} \\
& +\varepsilon K M\left\{\sum_{i=1}^{d_{r}+1} \int_{J_{r T, t}^{i}}\left\|\dot{x}_{i}^{(r-1)}\left(\Delta_{1}^{(r)}(\theta), \tau_{i-1}^{(r-1)}, x_{i-1}^{(r-1)+}\right)\right\| d 0\right. \\
& \left.+\int_{\tilde{J}_{r T, t}^{+}}\left\|\dot{x}_{1}^{(r)}\left(\Delta_{1}^{(r)}(\theta), r T, x_{r T}\right)\right\| d \theta\right\} \leq 2 \varepsilon^{2} K M^{3} \int_{r T}^{t} d \theta \int_{r T}^{\theta} d l \\
& +\varepsilon^{2} K M \sum_{i=1}^{d_{r}+1} \int_{J_{r T, t}^{i}}\left\{\int_{(r-1) T}^{\tau_{i-1}^{(r-1)}}\left\|A\left(l, x_{(r-1) T}, x_{(r-1) T}, 0\right)\right\| \cdot\left\|X\left(l, x_{(r-1) T}\right)\right\| d l\right. \\
& +\sum_{j=0}^{i-1}\left\|I_{j}^{(r-1)}\right\|+\varepsilon^{-1} \sum_{j=1}^{i-1}\left\|R_{j}^{(r-1)}\left(\tau_{j}^{(r-1)}, \tau_{j-1}^{(r-1)}, x_{j-1}^{(r-1)+}, \varepsilon\right)\right\| \\
& +\int_{\tau_{i-1}^{(r-1)}}^{\Delta_{1}^{(r)}(\theta)} \| A\left(l, x_{i}^{(r-1)}\left(l, \tau_{i-1}^{(r-1)}, x_{i-1}^{(r-1)+}\right), x_{i}^{(r-1)}\left(\Delta_{i}^{(r-1)}(l), \tau_{i-1}^{(r-1)}, x_{i-1}^{(r-1)+}\right),\right. \\
& \left.\left.\dot{x}_{i}^{(r-1)}\left(\Delta_{i}^{(r-1)}(l), \tau_{i-1}^{(r-1)}, x_{i-1}^{(r-1)+}\right)\right)\|\cdot\| X\left(l, x_{i}^{(r-1)}\left(l, \tau_{i-1}^{(r-1)}, x_{i-1}^{(r-1)+}\right)\right) \| d l\right\} d \theta \\
& +\varepsilon K M T\left[\left\|x_{r T}-B_{r} x_{(r-1) T}\right\|+\left\|B_{r} x_{(r-1) T}-x_{(r-1) T}\right\|\right] \\
& +\varepsilon^{2} K M^{3} \int_{\tilde{J}_{r T, t}^{+}}\left(\Delta_{1}^{(r)}(\theta)-r T\right) d \theta+\varepsilon^{2} K M\left\{\sum_{i=1}^{d_{r+1}} \int_{J_{r T, t}^{i}} \| A\left(\Delta_{1}^{(r)}(\theta),\right.\right. \\
& x_{i}^{(r-1)}\left(\Delta_{1}^{(r)}(\theta), \tau_{i-1}^{(r-1)}, x_{i-1}^{(r-1)+}\right), x_{i}^{(r-1)}\left(\Delta_{i}^{(r-1)}\left(\Delta_{1}^{(r)}(\theta)\right), \tau_{i-1}^{(r-1)}, x_{i-1}^{(r-1)+}\right), \\
& \left.\dot{x}_{i}^{(r-1)}\left(\Delta_{i}^{(r-1)}\left(\Delta_{1}^{(r)}(\theta)\right), \tau_{i-1}^{(r-1)}, x_{i-1}^{(r-1)+}\right)\right)\|\cdot\| X\left(\Delta_{1}^{(r)}(\theta),\right. \\
& \left.x_{i}^{(r-1)}\left(\Delta_{1}^{(r)}(\theta), \tau_{i-1}^{(r-1)}, x_{i-1}^{(r-1)+}\right)\right)\left\|d \theta+\int_{\tilde{J}_{r T, t}^{+}}\right\| A\left(\Delta_{1}^{(r)}(\theta), x_{1}^{(r)}\left(\Delta_{1}^{(r)}(\theta), r T, x_{r T}\right),\right. \\
& \left.x_{1}^{(r)}\left(\Delta_{1}^{(r)}\left(\Delta_{1}^{(r)}(\theta)\right), r T, x_{r T}\right), \dot{x}_{1}^{(r)}\left(\Delta_{1}^{(r)}\left(\Delta_{1}^{(1)}(0)\right), r T, x_{r T}\right)\right) \| \\
& \left.\cdot\left\|X\left(\Delta_{1}^{(r)}(\theta), x_{1}^{(r)}\left(\Delta_{1}^{(r)}(\theta), r T, x_{r T}\right)\right)\right\| d \theta\right\} \leq \varepsilon^{2} K M^{3} T^{2}
\end{aligned}
$$




$$
\begin{aligned}
& +\varepsilon^{2} K M^{2} \sum_{i=1}^{d_{r+1}+1} \int_{J_{r T, t}^{i}}\left\{M\left[\Delta_{1}^{(r)}(\theta)-(r-1) T\right]+(i-1)\right\} d \theta \\
& +\varepsilon K M \sum_{i=1}^{d_{r}+1}\left(\sum_{j=0}^{i-1} \omega_{j}^{(r-1)}\left(\varepsilon^{2}, T\right) \int_{J_{r T, t}^{i}} d \theta\right)+\varepsilon^{2} K M T\left[\alpha(T) T+\varepsilon M_{r}\right. \\
& +M(M+d) T]+\varepsilon^{2} K M^{3} \int_{r T}^{t}(\theta-r T) d \theta+\varepsilon^{2} K M^{3}\left(\sum_{i=1}^{d_{r+1}} \int_{J_{r T, t}^{i}} d \theta\right. \\
& \left.+\int_{\tilde{J}_{r T, t}^{+}} d \theta\right) \leq \varepsilon^{2} K M^{3} T^{2}+\varepsilon^{2} K M^{2} \int_{r T}^{t}\left\{M[\theta-(r-1) T]+d_{r}\right\} d \theta \\
& +\varepsilon K M T \sum_{j=0}^{d_{r}} \omega_{j}^{(r-1)}\left(\varepsilon^{2}, T\right)+\varepsilon^{2} K M T\left[\alpha(T) T+\varepsilon M_{r}\right. \\
& +M(M+d) T]+\varepsilon^{2} K M^{3} T^{2} / 2+\varepsilon^{2} K M^{3} T \leq 3 \varepsilon^{2} K M^{3} T^{2} / 2 \\
& +\varepsilon^{2} K M M^{2} T\left(2 M T+d_{r}\right)+\varepsilon^{2} K M^{2} T^{2}(M+d) \\
& +\varepsilon^{2} K M T\left[\alpha(T) T+\varepsilon M_{r}\right]+\varepsilon^{2} K M^{3} T \\
& +\varepsilon K M T \sum_{i=0}^{d_{r}} \omega_{i}^{(r-1)}\left(\varepsilon^{2}, T\right) \equiv \omega_{1}^{(r)}\left(\varepsilon^{2}, T\right),
\end{aligned}
$$

where

$$
\begin{aligned}
& \left(\bigcup_{i=1}^{d_{r+1}+1} J_{r T, t}^{i}\right) \cup \tilde{J}_{r T, t}^{+}=(r T, t], \\
& \left.J_{r T, t}^{i}=\left\{\theta: \theta \in(r T, t] \wedge \Delta_{1}^{(r)}(\theta) \in\left(\tau_{i-1}^{(r-1)}, \tau_{i}^{(r-1)}\right]\right\}, \quad i=\overline{1,\left(d_{r}+1\right.}\right) \\
& \tilde{J}_{r T, t}^{+}=(r T, t] \backslash\left(\bigcup_{i=1}^{d_{r}+1} J_{r T, t}^{i}\right), \\
& \left(\tau_{i}^{(0)} \equiv \tau_{i}, \quad i=\overline{0}, \overline{d_{1}}, \quad \tau_{d_{1}+1}^{(0)} \equiv T\right) .
\end{aligned}
$$

Therefore, the function $\widetilde{x}_{1}^{(r)}\left(t, r T, x_{r T}\right)$ approximates the solution of (22) on the interval $(r T,(r+1) T]$ to a precision of order $\varepsilon^{2}$.

For the root $\tau_{1}^{(r)}$ of the equation

$$
t=t_{d_{1}+\cdots+d_{r+1}}\left(x_{1}^{(r)}\left(t, r T, x_{r T}\right)\right)
$$

we obtain

$$
\tau_{1}^{(r)}=t_{1}^{(r)}+\varepsilon \Theta_{1}^{(r)}+O\left(\varepsilon^{2}\right),
$$

where

$$
\Theta_{1}^{(r)}=\frac{\partial \iota_{d_{1}+\cdots+a_{r}+1}}{\partial x}\left(x_{r T}\right) \int_{r T}^{t_{1}^{(r)}} A\left(0, x_{r T}, x_{r T}, 0\right) \mathrm{I}\left(\theta, x_{r T}\right) d 0 .
$$


(20) and (23) imply that for $\varepsilon$ sufficiently small the inequality $\tau_{1}^{(r)}>r T$ holds.

Thus

$$
x(t)=x_{1}^{(r)}\left(t, r T, x_{r T}\right)=\widetilde{x}_{1}^{(r)}\left(t, r T, x_{r T}\right)+R_{1}^{(r)}\left(t, r T, x_{r T}, \varepsilon\right)
$$

for $r T<t \leq \tau_{1}^{(r)}$.

Further on we obtain

$$
\begin{aligned}
x_{1}^{(r)+}= & x_{1}^{(r)}\left(\tau_{1}^{(r)}, r T, x_{r T}\right)+\varepsilon I_{d_{1}+\cdots+d_{r+1}}\left(x_{1}^{(r)}\left(\tau_{1}^{(r)}, r T, x_{r T}\right)\right) \\
= & \widetilde{x}_{1}^{(r)}\left(\tau_{1}^{(r)}, r T, x_{r T}\right)+\varepsilon I_{1}^{(r)}+R_{1}^{(r)}\left(\tau_{1}^{(r)}, r T, x_{r T}, \varepsilon\right) \\
= & x_{r T}+\varepsilon \int_{r T}^{\tau_{1}^{(r)}} A\left(\theta, x_{r T}, x_{r T}, 0\right) X\left(\theta, x_{r T}\right) d \theta \\
& +\varepsilon I_{1}^{(r)}+R_{1}^{(r)}\left(\tau_{1}^{(r)}, r T, x_{r T}, \varepsilon\right),
\end{aligned}
$$

where $I_{1}^{(r)} \equiv I_{d_{1}+\cdots+d_{r+1}}\left(x_{1}^{(r)}\left(\tau_{1}^{(r)}, r T, x_{r T}\right)\right)$

In the general case $s=\overline{2,\left(d_{r+1}+1\right)}$ we denote by $x_{s}^{(r)}\left(t, \tau_{s-1}^{(r)}, x_{s-1}^{(r)+}\right)$ the solution of the system

$$
x_{s}^{(r)}\left(t, \tau_{s-1}^{(r)}, x_{s-1}^{(r)+}\right)
$$

$$
\begin{aligned}
& =\left\{\begin{array}{c}
x_{s-1}^{(r)+}+\varepsilon \int_{\tau_{s-1}^{(r)}}^{t} A\left(\theta, x_{s}^{(r)}\left(\theta, \tau_{s-1}^{(r)}, x_{s-1}^{(r)+}\right),\right. \\
\left.x_{s}^{(r)}\left(\Delta_{s}^{(r)}(\theta), \tau_{s-1}^{(r)}, x_{s-1}^{(r)+}\right), \dot{x}_{s}^{(r)}\left(\Delta_{s}^{(r)}(\theta), \tau_{s-1}^{(r)}, x_{s-1}^{(r)+}\right)\right) \\
\cdot X\left(\theta, x_{s}^{(r)}\left(\theta, \tau_{s-1}^{(r)}, x_{s-1}^{(r)+}\right)\right) d \theta, \quad t>\tau_{s-1}^{(r)}, \\
x_{s-1}^{(r)}\left(t, \tau_{s-2}^{(r)}, x_{s-2}^{(r)+}\right), \quad-\delta \leq t \leq \tau_{s-1}^{(r)},
\end{array}\right. \\
& \dot{x}_{s}^{(r)}\left(t, \tau_{s-1}^{(r)}, x_{s-1}^{(r)+}\right)=\dot{x}_{s-1}^{(r)}\left(t, \tau_{s-2}^{(r)}, x_{s-2}^{(r)+}\right), \quad-\delta \leq t \leq \tau_{s-1}^{(r)},
\end{aligned}
$$

where

$$
\Delta_{s}^{(r)}(t)=\Delta\left(t, x_{s}^{(r)}\left(t, \tau_{s-1}^{(r)}, x_{s-1}^{(r)+}\right)\right)
$$

and

$$
\begin{aligned}
x_{s-1}^{(r)+}= & x_{s-1}^{(r)}\left(\tau_{s-1}^{(r)}, \tau_{s-2}^{(r)}, x_{s-2}^{(r)+}\right)+\varepsilon I_{d_{1}+\cdots+d_{r+s-1}}\left(x_{s-1}^{(r)}\left(\tau_{s-1}^{(r)}, \tau_{s-2}^{(r)}, x_{s-2}^{(r)+}\right)\right) \\
= & x_{r T}+\varepsilon \int_{r T}^{\tau_{s-1}^{(r)}} A\left(\theta, x_{r T}, x_{r T}, 0\right) X\left(\theta, x_{r T}\right) d \theta \\
& +\varepsilon \sum_{i=1}^{s-1} I_{i}^{(r)}+\sum_{i=1}^{s-1} R_{i}^{(r)}\left(\tau_{i}^{(r)}, \tau_{i-1}^{(r)}, x_{i-1}^{(r)+}, \varepsilon\right), \\
I_{s-1}^{(r)} \equiv & I_{d_{1}+\cdots+d_{r}+s-1}\left(x_{s-1}^{(r)}\left(\tau_{s-1}^{(r)}, \tau_{s-2}^{(r)}, x_{s-2}^{(r)+}\right)\right) .
\end{aligned}
$$


The solution of (24) coincides with the solution of the system of functional-differential equations (1) with impulses on the interval $[-\delta$, $\left.\tau_{s}^{(r)}\right]$.

We consider the function

$$
\widetilde{x}_{s}^{(r)}\left(t, \tau_{s-1}^{(r)}, x_{s-1}^{(r)+}\right)=x_{s-1}^{(r)+}+\varepsilon \int_{\tau_{s-1}^{(r)}}^{t} A\left(\theta, x_{r T}, x_{r T}, 0\right) X\left(\theta, x_{r T}\right) d \theta .
$$

It can be shown that on the interval $r T<\tau_{s-1}^{(r)}<t \leq(r+1) T$ the following estimate holds

$$
\begin{aligned}
& \left\|R_{s}^{(r)}\left(t, \tau_{s-1}^{(r)}, x_{s-1}^{(r)+}, \hat{\varepsilon}\right)\right\| \\
& =\left\|x_{s}^{(r)}\left(t, \tau_{s-1}^{(r)}, x_{s-1}^{(r)+}\right)-\widetilde{x}_{s}^{(r)}\left(t, \tau_{s-1}^{(r)}, x_{s-1}^{(r)+}\right)\right\| \\
& \leq 3 \varepsilon^{2} K M[M T+(s-1)]^{2} / 2+\varepsilon^{2} K M^{2} T\left(2 M T+d_{r}\right) \\
& +\varepsilon^{2} K M^{2} T^{2}(M+d)+\varepsilon^{2} K M T\left[\alpha(T) T+\varepsilon M_{r}\right] \\
& +\varepsilon^{2} K M^{3} T+\varepsilon K M T \sum_{i=0}^{d_{r}} \omega_{i}^{(r-1)}\left(\varepsilon^{2}, T\right) \\
& +3 \varepsilon K M T \sum_{i=1}^{s-1} \omega_{i}^{(r)}\left(\varepsilon^{2}, T\right) \equiv \omega_{s}^{(r)}\left(\varepsilon^{2}, T\right) .
\end{aligned}
$$

Since

$$
\begin{aligned}
& \widetilde{x}_{s}^{(r)}\left(t, \tau_{s-1}^{(r)}, x_{s-1}^{(r)+}\right)=x_{r T}+\varepsilon \int_{r T}^{t} A\left(\theta, x_{r T}, x_{r T}, 0\right) X\left(\theta, x_{r T}\right) d \theta \\
& \quad+\varepsilon \sum_{i=1}^{s-1} I_{i}^{(r)}+\sum_{i=1}^{s-1} R_{i}^{(r)}\left(\tau_{i}^{(r)}, \tau_{i-1}^{(r)}, x_{i-1}^{(r)+}, \varepsilon\right) \\
& =\widetilde{x}_{1}^{(r)}\left(t, r T, x_{r T}\right)+\varepsilon \sum_{i=1}^{s-1} I_{i}^{(r)}+\sum_{i=1}^{s-1} R_{i}^{(r)}\left(\tau_{i}^{(r)}, \tau_{i-1}^{(r)}, x_{i-1}^{(r)}, \varepsilon\right),
\end{aligned}
$$

then we obtain

$$
\begin{aligned}
x(t)= & x_{s}^{(r)}\left(t, \tau_{s-1}^{(r)}, x_{s-1}^{(r)+}\right)=\widetilde{x}_{1}^{(r)}\left(t, r T, x_{r T}\right) \\
& +\varepsilon \sum_{i=0}^{s-1} I_{i}^{(r)}+\sum_{i=0}^{s-1} R_{i}^{(r)}\left(\tau_{i}^{(r)}, \tau_{i-1}^{(r)}, x_{i-1}^{(r)+}, \varepsilon\right)+R_{s}^{(r)}\left(t, \tau_{s-1}^{(r)}, x_{s-1}^{(r)+}, \varepsilon\right)
\end{aligned}
$$

for

$$
t_{s-1}^{(r)}+\varepsilon \Theta_{s-1}^{(r)}+\gamma_{s-1} O\left(\varepsilon^{2}\right)=\tau_{s-1}^{(r)}<t \leq \tau_{s}^{(r)}+\varepsilon \Theta_{s}^{(r)}+O\left(\varepsilon^{2}\right)
$$

where

$$
\Theta_{s}^{(r)}=\frac{\partial t_{d_{1}+\cdots+d_{r}+s}\left(x_{r T}\right)}{\partial x}\left[\int_{r T}^{t_{s}^{(r)}} A\left(\theta, x_{r T}, x_{r T}, 0\right) X\left(\theta, x_{r T}\right) d \theta\right.
$$




$$
\begin{aligned}
& \left.+\sum_{i=0}^{s-1} I_{i}^{(r)}\right], \quad t_{0}^{(r)}=r T, \quad \Theta_{0}^{(r)}=\gamma_{0}=0, \quad I_{0}^{(r)}=0, \\
& R_{0}^{(r)}\left(\tau_{0}^{(r)}, \tau_{-1}^{(r)}, x_{-1}^{(r)+}, \varepsilon\right)=0, \quad \gamma_{s}=1, \quad s=1, \bar{d}_{r+1},
\end{aligned}
$$

as well as for

$$
\begin{aligned}
t_{d_{r+1}}^{(r)}+\varepsilon \Theta_{d_{r+1}}^{(r)}+O\left(\varepsilon^{2}\right) & =\tau_{d_{r+1}}^{(r)}<t \leq \tau_{d_{r+1}+1}^{(r)}=(r+1) T \\
s & =d_{r+1}+1
\end{aligned}
$$

We work out $x((r+1) T)$ and $\bar{x}((r+1) T)$

$$
\begin{aligned}
& x((r+1) T)=x_{d_{r+1}+1}^{(r)}\left((r+1) T, \tau_{d_{r+1}}^{(r)}, x_{d_{r+1}}^{(r)+}\right) \\
& =x_{r T}+\varepsilon \int_{r T}^{(r+1) T} A\left(\theta, x_{r T}, x_{r T}, 0\right) X\left(\theta, x_{r T}\right) d \theta+\varepsilon \sum_{i=0}^{d_{r+1}} I_{i}^{(r)} \\
& \quad+\sum_{i=0}^{d_{r+1}+1} R_{i}^{(r)}\left(\tau_{i}^{(r)}, \tau_{i-1}^{(r)}, x_{i-1}^{(r)+}, \varepsilon\right)=x_{r T}+\varepsilon I_{0}\left(x_{r T}\right) T \\
& \quad+\varepsilon A_{0}\left(x_{r T}\right) \int_{r T}^{(r+1) T} X\left(\theta, x_{r T}\right) d \theta \\
& \quad+\varepsilon \int_{r T}^{(r+1) T}\left[A\left(\theta, x_{r T}, x_{r T}, 0\right)-A_{0}\left(x_{r T}\right)\right] X\left(\theta, x_{r T}\right) d \theta \\
& \quad+\varepsilon\left[\sum_{i=0}^{d_{r+1}} I_{i}^{(r)}-I_{0}\left(x_{r T}\right) T\right]+\sum_{i=0}^{d_{r+1}+1} R_{i}^{(r)}\left(\tau_{i}^{(r)}, \tau_{i-1}^{(r)}, x_{i-1}^{(r)+}, \varepsilon\right), \\
& \bar{x}((r+1) T)=x_{0}+\varepsilon \int_{0}^{(r+1) T}\left[A_{0}(\bar{x}(\theta)) X(\theta, \bar{x}(\theta))+I_{0}(\bar{x}(\theta))\right] d \theta \\
& =\bar{x}(r T)+\varepsilon \int_{r T}^{(r+1) T}\left[A_{0}(\bar{x}(\theta)) X(\theta, \bar{x}(\theta))+I_{0}(\bar{x}(\theta))\right] d \theta .
\end{aligned}
$$

Taking into consideration the definition of the operator $B_{r+1}$ and the results for $x((r+1) T)$ and $\bar{x}((r+1) T)$ we can write

$$
\begin{aligned}
& \|x((r+1) T)-\bar{x}((r+1) T)\| \leq\left\|x((r+1) T)-B_{r+1} x_{r T}\right\| \\
& \quad+\left\|B_{r+1} x_{r T}-B_{r+1} \bar{x}(r T)\right\|+\left\|B_{r+1} \bar{x}(r T)-\bar{x}((r+1) T)\right\| .
\end{aligned}
$$

Dealing in a similar way as in (17), for the first addend on the right-hand side of (27) we get

$$
\begin{aligned}
& \left\|x((r+1) T)-B_{r+1} x_{r T}\right\| \\
& \leq \varepsilon \alpha(T) T+\varepsilon^{2} K M d_{r+1}\left(2 M T+d_{r+1}-1\right) / 2 \\
& \quad+\sum_{i=0}^{d_{r+1}+1} \omega_{i}^{(r)}\left(\varepsilon^{2}, T\right)+\varepsilon K \sum_{i=1}^{d_{r+1}} \sum_{l=0}^{i} \omega_{l}^{(r)}\left(\varepsilon^{2}, T\right)
\end{aligned}
$$




$$
=\varepsilon \alpha(T) T+\varepsilon^{2} M_{r+1},
$$

where $\omega_{0}^{(r)}\left(\varepsilon^{2}, T\right) \equiv 0, M_{r \perp 1}=M_{r\lrcorner 1}\left(T, d_{1}, \cdots, d_{r \perp 1}\right)$ is a constant.

For the second addend on the right-hand side of (27) we have

$$
\begin{aligned}
& \left\|B_{r+1} x_{r T}-B_{r+1} \bar{x}(r T)\right\|=\| x_{r T}+\varepsilon I_{0}\left(x_{r T}\right) T \\
& +\varepsilon A_{0}\left(x_{r T}\right) \int_{r T}^{(r+1) T} X\left(\theta, x_{r T}\right) d \theta-\bar{x}(r T)-\varepsilon I_{0}(\bar{x}(r T)) T \\
& +\varepsilon A_{0}(\bar{x}(r T)) \int_{r T}^{(r+1) T} X(\theta, \bar{x}(r T)) d \theta\|\leq\| x_{r T}-\bar{x}(r T) \| \\
& +\varepsilon T\left\|I_{0}\left(x_{r T}\right)-I_{0}(\bar{x}(r T))\right\|+\varepsilon\left\|A_{0}\left(x_{r T}\right)-A_{0}(\bar{x}(r T))\right\| . \\
& \int_{r T}^{(r+1) T}\left\|X\left(\theta, x_{r T}\right)\right\| d \theta \\
& \quad+\varepsilon\left\|A_{0}(\bar{x}(r T))\right\| \int_{r T}^{(r+1) T}\left\|X\left(\theta, x_{r T}\right)-X(\theta, \bar{x}(r T))\right\| d \theta \\
& \leq[1+\varepsilon(3 M+d) K T]\left\|x_{r T}-\bar{x}(r T)\right\| \\
& \leq[1+\varepsilon(3 M+d) K T] \sum_{i=0}^{r-1}[1+\varepsilon(3 M+d) K T]^{i} \\
& \quad \cdot\left[\varepsilon \alpha(T) T+\varepsilon^{2} \bar{M}\right] .
\end{aligned}
$$

where $\bar{M}=(M+d)(3 M+d) K M T^{2}+\max _{i=\overline{1, r}} M_{i}$

Since for $t \in(r T,(r+1) T]$ the inequality

$$
\begin{gathered}
\|\bar{x}(t)-\bar{x}(r T)\| \leq \varepsilon \int_{r T}^{t}\left[\left\|A_{0}(\bar{x}(\theta))\right\| \cdot\|X(\theta, \bar{x}(\theta))\|\right. \\
\left.+\left\|I_{0}(\bar{x}(\theta))\right\|\right] d \theta \leq \varepsilon(M+d) M T,
\end{gathered}
$$

holds, then for the third addend on the right-hand side of (27) we obtain

$$
\begin{aligned}
& \left\|B_{r+1} \bar{x}(r T)-\bar{x}((r+1) T)\right\|=\| \bar{x}(r T)+\varepsilon I_{0}(\bar{x}(r T)) T \\
& \quad+\varepsilon A_{0}(\bar{x}(r T)) \int_{r T}^{(r+1) T} X(\theta, \bar{x}(r T)) d \theta-\bar{x}(r T) \\
& \quad-\varepsilon \int_{r T}^{(r+1) T}\left[A_{0}(\bar{x}(\theta)) X(\theta, \bar{x}(\theta))+I_{0}(\bar{x}(\theta))\right] d \theta \| \\
& \leq \varepsilon\left\|A_{0}(\bar{x}(r T))\right\| \int_{r T}^{(r+1) T}\|X(\theta, \bar{x}(r T))-X(\theta, \bar{x}(\theta))\| d \theta \\
& \quad+\varepsilon \int_{r T}^{(r+1) T}\left\|A_{0}(\bar{x}(r T))-A_{0}(\bar{x}(\theta))\right\| \cdot\|X(\theta, \bar{x}(\theta))\| d \theta
\end{aligned}
$$




$$
\begin{aligned}
&+\varepsilon \int_{r T}^{(r+1) T}\left\|I_{0}(\bar{x}(r T))-I_{0}(\bar{x}(\theta))\right\| d \theta \\
& \leq \varepsilon(3 M+d) K \int_{r T}^{(r+1) T}\|\bar{x}(r T)-\bar{x}(\theta)\| d \theta \\
& \leq \varepsilon^{2}(M+d)(3 M+d) K M T^{2} . \\
&(27)-(30) \text { imply the inequality } \\
&\|x((r+1) T)-\bar{x}((r+1) T)\| \\
& \quad \leq \sum_{i=0}^{r}[1+\varepsilon(3 M+d) K T]^{i} \cdot\left[\varepsilon \alpha(T) T+\varepsilon^{2} \bar{M}\right],
\end{aligned}
$$

where $\quad \bar{M}=(M+d)(3 M+d) K M T^{2}+\max _{i=\overline{1,(r+1)}} M_{i}$

The last inequality shows that (7) is fulfilled for $p=r+1$ and that $x((r+1) T)$ belongs to the domain $D$.

Thus Lemma 1 is proved.

Proof of Theorem 1. By virtue of the condition $3^{\circ}$ of Theorem there exists a constant $C(T)<\infty$ such that for each $i=1,2, \cdots$ the inequality $d_{i}<C(T)$ holds. Hence, there also exists a constant $M_{0}(T)$ $<\infty$ such that

$$
\bar{M}=(M+d)(3 M+d) K M T^{2}+\max _{i=1,2, \cdots} M_{i} \leq M_{0}(T) .
$$

Let $q$ be equal to the whole part of the number $L / \varepsilon T$. Then for each $p \in \overline{1, q}$, by virtue of (31) and Lemma 1 , we have

$$
\begin{aligned}
& \|x(p T)-\bar{x}(p T)\| \\
& \quad \leq \varepsilon \sum_{i=0}^{p-1}[1+\varepsilon(3 M+d) K T]^{i}\left[\alpha(T) T+\varepsilon M_{0}(T)\right] \\
& \quad \leq\left[\alpha(T) T+\varepsilon M_{0}(T)\right][1+\varepsilon(3 M+d) K T]^{p} /(3 M+d) K T \\
& \quad \leq\left[e^{(3 M+d) K L}+O(\varepsilon)\right]\left[\alpha(T) T+\varepsilon M_{0}(T)\right] /(3 M+d) K T .
\end{aligned}
$$

We choose $T$ sufficiently large, so that

$$
e^{(3 M+d) K L} \alpha(T) /(3 M+d) K<\eta / 4
$$

and then we choose $\varepsilon$ sufficiently small, so that

$$
\left\{O(\varepsilon)\left[\alpha(T) T+\varepsilon M_{0}(T)\right]+\varepsilon e^{(3 M+d) K L} M_{0}(T)\right\} /(3 M+d) K T<\eta / 4 .
$$


Then for each $p \equiv \overline{1 . q}$ the following inequality will hold

$$
\left\|\cdot r\left(\rho^{\prime}\right) \quad x(p T)\right\| \cdot \cdots / 2 .
$$

Further on we estimate $\|\bar{x}(t)-\bar{x}((p-1) T)\|$ and $\| x(t)-$ $x((p-1) T) \|$ on the interval $(p-1) T \leq t \leq p T$.

We have

$$
\begin{aligned}
\| \bar{x}(t) & -\bar{x}((p-1) T) \| \\
& \leq \varepsilon \int_{(p-1) T}^{t}\left\|A_{0}(\bar{x}(\theta)) X(\theta, \bar{x}(\theta))+I_{0}(\bar{x}(\theta))\right\| d \theta \\
& \leq \varepsilon(M+d) M T \\
\| x(t) & -x((p-1) T)\|=\| x_{s}^{(p-1)}\left(t, \tau_{s-1}^{(p-1)}, x_{s-1}^{(p-1)+}\right)-x((p-1) T) \| \\
= & \| \widetilde{x}_{1}^{(p-1)}\left(t,(p-1) T, x_{(p-1) T}\right)+\varepsilon \sum_{i=0}^{s-1} I_{i}^{(p-1)} \\
& +\sum_{i=0}^{s-1} R_{i}^{(p-1)}\left(\tau_{i}^{(p-1)}, \tau_{i-1}^{(p-1)}, x_{i-1}^{(p-1)+}, \varepsilon\right)+R_{s}^{(p-1)}\left(t, \tau_{s-1}^{(p-1)}, x_{s-1}^{(p-1)+}, \varepsilon\right) \\
& -x((p-1) T) \| \\
\leq & \varepsilon \int_{(p-1) T}^{t}\left\|A\left(\theta, x_{(p-1) T^{\prime}}, x_{(p-1) T}, 0\right)\right\|\left\|X\left(\theta, x_{(p-1) T}\right)\right\| d \theta \\
& +\varepsilon \sum_{i=0}^{s-1}\left\|I_{i}^{(p-1)}\right\|+\sum_{i=0}^{s-1}\left\|R_{i}^{(p-1)}\left(\tau_{i}, \tau_{i-1}^{(p-1)}, x_{i-1}^{(p-1)+}, \varepsilon\right)\right\| \\
& +\left\|R_{s}^{(p-1)}\left(t, \tau_{s-1}^{(p-1)}, x_{s-1}^{(p-1)+}, \varepsilon\right)\right\| \leq \varepsilon M[M T+(s-1)] \\
& +\sum_{i=0}^{s} \omega_{i}^{(p-1)}\left(\varepsilon^{2}, T\right) \leq \varepsilon M[M T+C(T)]+\varepsilon^{2} M_{0}(T) \equiv \Psi(\varepsilon, T)
\end{aligned}
$$

We see that for $T$ chosen as it was, if $\varepsilon$ is sulficiently small, we shall have

$$
\Psi(\varepsilon, T)<\eta / 2 .
$$

It follows from (32)-(35) that for $T$ chosen as it was, if $\varepsilon$ is sufficiently small, for $p=1,2, \cdots, q$ on the interval $(p-1) T \leq t \leq p T$ the following inequality will hold

$$
\begin{aligned}
& \|x(t)-\bar{x}(t)\| \leq\|x(t)-x((p-1) T)\| \\
& \quad+\|x((p-1) T)-\bar{x}((p-1) T)\|+\|\bar{x}((p-1) T)-\bar{x}(t)\|<\eta .
\end{aligned}
$$


Therefore, for $T$ chosen as it was, if $\varepsilon$ is sufficiently small $(0<\varepsilon$ $\left.\leq \varepsilon_{0} \leq \mathcal{E}\right)$, the inequality $\|x(t)-\bar{x}(t)\|<\eta$ will hold on the whole interval $0 \leq t \leq L \varepsilon^{-1}$.

Thus Theorem 1 is proved.

\section{References}

[1] Mitropol'skii, Ju. A., The averaging method in non-linear mechanics, "Naukova Dumka", Kiev, 1971 (in Russian).

[2] Mitropol'skii, Ju. A. and Fodchuk, V. I., The asymptotic methods of non-linear mechanics applied to non-linear differential equations with time lag, Ukrain. Mat.Z., 18 (1966), 65-84 (in Russian).

[3] Mitropol'skii, Ju. A., Bainov, D. D. and Milusheva, S. D., Application of the averaging method for the solution of boundary-value problems for ordinary differential and integral-differential equations, Matematicheskaya fizika, 25 (1978), 3-22 (in Russian).

[4] Samoilenko, A. M., Application of the averaging method for studying oscillations induced by instantaneous impulses in self-oscillation systems of second order with a small parameter, Ukrain. Mat. Z., 13 (1961), 103-108 (in Russian).

[5] - On the justification of the averaging method for studying oscillations in systems undergoing impulse effect, Ukrain, Mat. Z. 19 (1967), 96-104 (in Russian).

[6] Samoilenko, A. M., Bainov, D. D. and Milusheva, S. D., On the application of the averaging method for systems of integro-differential equations of standard type with discontinuous right-hand side, Acta Math. Acad. Sci. Hungar., 29 (1977), 31-48.

[7] Bainov, D. D. and Milusheva, S. D., Justification of the averaging method for a system of differential equations with fast and slow variables and with impulses, Journal Applied Mathematics and Physics (ZAMP), 32 (1981), 237-254. 